\title{
IDEALS IN BIRKHOFF LATTICES
}

\author{
BY \\ R. P. DILWORTH(1)
}

Introduction. In previous papers by the author (Dilworth $[1,2])(2)$ methods were developed for studying the arithmetical properties of Birkhoff lattices, that is, the properties of irreducibles and decompositions into irreducibles. These methods, however, required the assumption of both the ascending and descending chain conditions. In this paper we give a new technique which is applicable in general and which under the assumption of merely the ascending chain condition gives results quite as good as those of the previous work. Now the descending chain condition is equivalent to the requirement that every ideal $\left({ }^{3}\right)$ be principal. Hence if the descending chain condition does not hold we find it convenient to relate the arithmetical properties of the lattice to the structure of its lattice of ideals. Furthermore since the Birkhoff condition itself may lose much of its force if the descending chain condition does not hold, a lattice is defined to be a Birkhoff lattice if every element satisfies the Birkhoff condition $\left({ }^{4}\right)$ in the lattice of ideals. Hence if the descending chain condition holds, this definition reduces to that used in the previous papers. In the lattice of ideals, the existence of sufficient covering ideals to make the Birkhoff conditions effective can be proved.

In D1 and D2 it was shown that the arithmetical behavior of an element $a$ was closely related to the structure of the quotient lattice $\mathfrak{S}_{a}$ generated by the elements covering $a$. Here we make a similar correlation with the structure of the quotient lattice of ideals $\mathfrak{R}_{a}$ generated by the ideals covering $a$. The important properties of $\mathfrak{S}_{a}$ follow from its finite dimensionality. $\mathfrak{R}_{a}$ on the other hand is in general not finite dimensional and thus one of the essential problems of the present treatment is the proof of the archimedean character of $\mathfrak{R}_{a}$ in the cases of interest.

If the descending chain condition holds, the Birkhoff condition is equivalent to Mac Lane's point-free exchange axiom $E_{5}$ (Mac Lane [1]). Now $E_{5}$ is independent of covering conditions, which suggests that it should be closely related to the Birkhoff condition in the lattice of ideals. We show that the Birkhoff condition in the lattice of ideals always implies $E_{5}$ and, if each principal ideal is covered by only a finite number of ideals, the two conditions are equivalent.

Presented to the Society, December 27, 1939; received by the editors May 11, 1940.

(1) Sterling Research Fellow, Yale University.

(2) These papers will be referred to as D1 and D2.

(3) An ideal is a sublattice which contains with each element all of its divisors. G. Birkhoff (Birkhoff [1]) uses the term dual ideal for such a sublattice.

(4) See $\$ 1$, Conditions B1 and B1'. 
In $\mathrm{D} 1$ it was shown that a lattice of finite dimensions has unique irreducible decompositions if and only if it is a Birkhoff lattice in which every modular sublattice is distributive. This result no longer holds if we drop the descending chain condition as we show by an example. However, by strengthening slightly the condition that every modular sublattice be distributive, we have the following theorem:

THEOREM 6.6. Let $\mathfrak{S}$ satisfy the ascending chain condition. Then every element of $\subseteq$ is uniquely expressible as a reduced crosscut of irreducibles if and only if the following conditions hold.

$\mathrm{E}_{5}$. (Mac Lane's point-free exchange axiom.) $a \supset b, \supset a \cap c, c \neq a \cap c$ implies that $c_{1} \neq a \cap c$ exists such that $c \supset c_{1} \supset a \cap c$ and $b=a \cap\left(b \cup c_{1}\right)$.

A. $a \cup b \supset x \supset a \cap b, a \cap x=b \cap x=a \cap b$ implies $x=a \cap b$.

If we go over to the lattice of ideals, $E_{5}$ may be replaced by the condition that $\subseteq$ be a Birkhoff lattice, and $\mathrm{A}$, by the requirement that the ideals covering a principal ideal generate a Boolean algebra.

In D2, Birkhoff lattices in which the number of components in the irreducible decompositions of each element is unique were characterized in terms of the structure of the quotient lattices $\Im_{a}$. We prove here:

THEOREM 5.1. Let $\subseteq$ be a Birkhoff lattice satisfying the ascending chain condition and let $\mathfrak{R}$ denote its lattice of ideals. Then the number of components in the irreducible decompositions of each element of the lattice $\subseteq$ is unique if and only if the ideals covering any principal ideal of the lattice $\mathfrak{R}$ generate a dense, modular sublattice of $\mathbb{R}$.

By means of ideal methods we give a new proof of the Kurosch-Ore decomposition theorem for modular lattices in its most general form. The proof rests on the fact that if an element of a modular lattice has a decomposition into irreducibles then the sublattice generated by the ideals covering the element is of finite dimensions.

Finally $\S \S 7$ and 8 contain examples which show the complications which may arise when the descending chain condition does not hold.

1. Notation and definitions. The fixed lattice of elements $a, b, c, \cdots$ will

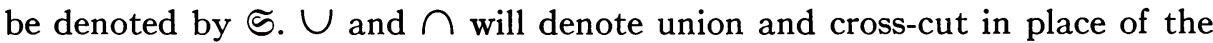
symbols $($,$) and [$,$] used in D1 and D2. \supset$ denotes lattice division. $a=b$ is defined by the two formulas $a \supset b, b \supset a$. If $a \supset b, a \neq b$ and $a \supset x \supset b$ implies $a=x$ or $x=b$, we say that $a$ covers $b$ and write $a>b$. Elements which cover the null element $z$ of a lattice are called points and elements covered by the unit element $u$ are said to be simple.

A lattice $\subseteq$ satisfies the ascending (descending) chain condition if every chain $a_{1} \subset a_{2} \subset a_{3} \subset \cdots\left(a_{1} \supset a_{2} \supset a_{3} \supset \cdots\right)$ has only a finite number of distinct elements. If both the ascending and descending chain conditions hold, $\checkmark$ is said to be archimedean or of finite dimensions. 
Throughout the paper we shall be particularly interested in lattices which satisfy the following weak form of the modular axiom.

B1. $a>a \cap b \rightarrow a \cup b>b\left(^{5}\right)$.

Another form of $\mathrm{B} 1$ is the following:

$\mathrm{B} 1^{\prime} . b>a, c \supset a, c D b \rightarrow b \cup c>c$.

If $\mathrm{B} 1^{\prime}$ is satisfied for a given $a$ and any $b$ and $c$ we say that $a$ satisfies the Birkhoff condition in $\mathfrak{S}$. Hence B1 holds in $\subseteq$ if and only if each element of $\mathfrak{S}$ satisfies the Birkhoff condition.

We state now some lemmas on elements satisfying the Birkhoff condition which are refinements of Lemmas 3.1-3.3 of D2.

LemMA 1.1. Let a satisfy the Birhoff condition in $\mathfrak{\subseteq}$ and let $a_{1}, \cdots, a_{k}>a$. Then each union independent $\left({ }^{6}\right)$ set of the $a_{i}$ is contained in a maximal independent set.

The usual proof is valid under the weaker hypotheses of the lemma.

LEMmA 1.2. Let a satisfy the Birkhoff condition and let $a_{1}, \cdots, a_{k}>a$. Then each union independent set of the $a_{i}$ generates a Boolean algebra.

We note that the usual proof (for example Theorem 2.3 of D1) is not valid in this case since it depends upon the existence of a rank function. Under the hypotheses of the lemma, complete chains need not have the same length and hence a rank function will in general not exist.

Now let $A$ and $B$ be two arbitrary subsets of the set $\left\{a_{1}, \cdots, a_{k}\right\}$. Let $\Sigma(A)$ denote the union of the elements of $A$ and denote the set-theoretic union and cross-cut of $A$ and $B$ by $A \cup B$ and $A \cap B$ respectively. We shall show that

$$
\Sigma(A) \cap \Sigma(B)=\Sigma(A \cap B) .
$$

Let $\mu(A)$ denote the number of elements in $A$ and set $\nu(A)=k-\mu(A)$. If $\nu(A \cap B)=0$, then $\mu(A \cap B)=k$ and $A=B$. Hence (1) holds. If $\nu(A \cap B)=1$, then either $A \supset B$ or $B \supset A$ and again (1) holds. Now let (1) hold for all $A$ and $B$ such that $\nu(A \cap B)<l$. Let $\nu(A \cap B)=l$ for some $A$ and $B$. Then $\mu(A \cap B)=k-l=r$. Hence $A=\left\{a_{1}, \cdots, a_{r}, a_{r+1}, \cdots, a_{s}\right\}$ and $B=\left\{a_{1}, \cdots\right.$, $\left.a_{r}, a_{r+1}^{\prime}, \cdots, a_{t}^{\prime}\right\}$. Since (1) is trivial if $B \supset A$, we may assume that $s>r$. Let $B^{\prime}=\left\{a_{1}, \cdots, a_{r}, a_{r+1}, a_{r+1}^{\prime}, \cdots, a_{t}^{\prime}\right\}$. Now $\mu\left(A \cap B^{\prime}\right)=r+1$ and hence $\nu\left(A \cap B^{\prime}\right)=k-(r+1)=l-1<l$. By the induction assumption $\Sigma\left(A \cap B^{\prime}\right)$ $=\Sigma(A) \cap \Sigma\left(B^{\prime}\right)$. Thus $\Sigma\left(A \cap B^{\prime}\right)=\Sigma(A) \cap \Sigma\left(B^{\prime}\right) \supset \Sigma(A) \cap \Sigma(B) \supset \Sigma(A \cap B)$.

(5) $\rightarrow$ denotes formal implication.

(6) A set of elements $x_{1}, \cdots, x_{n}$ is said to be union independent or simply independent if $x_{1} \cup \ldots \cup \cup_{x_{i-1}} \cup_{x_{i+1}} \cup \ldots \cup x_{n} D_{x_{i}}, i=1, \ldots, n$. Similarly the set is said to be cross-cut independent if $x_{i} D x_{1} \cap \cdots \cap x_{i-1} \cap x_{i+1} \cap \cdots \cap x_{n}, i=1, \cdots, n$. 
Since $a_{1}, \cdots, \quad a_{k}$ are independent we have $\Sigma(A \cap B) D a_{r+1}$ and hence $\Sigma\left(A \cap B^{\prime}\right)=a_{r+1} \cup \Sigma(A \cap B)>\Sigma(A \cap B)$. If $\Sigma\left(A \cap B^{\prime}\right)=\Sigma(A) \cap \Sigma(B)$, then $\Sigma(B) \supset a_{r+1}$ contrary to the independence of $a_{1}, \cdots, a_{k}$. Hence $\Sigma(A) \cap \Sigma(B)$ $=\Sigma(A \cap B)$. Thus (1) holds for $\nu(A \cap B)=l$ and by induction (1) holds for all $A$ and $B$. Clearly $\Sigma(A) \cup \Sigma(B)=\Sigma(A \cup B)$. If $\Sigma(A)=\Sigma(B)$, then $A=B$ by the independence of $a_{1}, \cdots, a_{k}$. Hence the elements which can be expressed as a union of the $a_{i}$ are isomorphic to the subsets of $a_{1}, \cdots, a_{k}$ under union and cross-cut and thus $a_{1}, \cdots, a_{k}$ generate a Boolean algebra. This completes the proof of the lemma.

Lemma 1.3. Let a satisfy the Birkhoff condition and let $a_{1}, \cdots, a_{k}>a$. Then any two maximal union independent sets of the $a_{i}$ have the same number of elements and any element of one set may be replaced by a suitably chosen element of the other without altering the maximal property.

The usual proof is valid in this case.

LEMMA 1.4. Let a satisfy the Birkhoff condition and let $a_{1}, \cdots, a_{k}>a$. Then any chain joining $a_{1} \cup \cdots \cup a_{k}$ to a has not more than $k+1$ distinct members.

We may clearly suppose that $a_{1}, \cdots, a_{k}$ are independent. Let $a=b_{0} \subset b_{1}$ $\subset b_{2} \subset \ldots \subset b_{l-1} \subset b_{l}=a_{1} \cup \ldots \cup a_{k}$ be a chain joining $a_{1} \cup \ldots \cup a_{k}$ to $a$ having $l+1$ distinct members and let us assume that $l>k$. Clearly $b_{0}<b_{0} \cup a_{1}$ $<\cdots<b_{0} \cup a_{1} \cup \ldots \cup a_{k-1}<a_{1} \cup \ldots \cup a_{k}$ by the Birkhoff condition. Now suppose that it has been shown that $a \subset b_{1} \subset \cdots \subset b_{i}<b_{i} \cup a_{1}<\cdots<b_{i} \cup a_{1}$ $\cup \ldots \cup a_{k_{i}-1}<a_{1} \cup \ldots \cup a_{k}$ where $k_{i} \leqq k-i$ and $i<k$. Consider the chain $a \subset b_{1} \subset \cdots \subset b_{i} \subset b_{i+1} \subset b_{i+1} \cup a_{1} \subset \ldots \subset b_{i+1} \cup a_{1} \cup \ldots \cup a_{k_{i}-1} \subset a_{1} \cup \ldots \cup a_{k}$. Let us assume that all of the members of this chain are distinct. If $b_{i} \cup a_{1} \cup \ldots \cup a_{k_{i}-1} \nsupseteq b_{i+1}$, then $a_{1} \cup \ldots \cup a_{k} \supset e_{i+1} \cup a_{1} \cup \ldots \cup a_{k_{i-1}} \supset b_{i} \cup a_{1}$ $\cup \ldots \cup a_{k_{i}-1}$ and $b_{i+1} \cup a_{1} \cup \ldots \cup a_{k_{i}-1} \neq b_{i} \cup a_{i} \cup \ldots \cup a_{k_{i-1}}$. But $a_{1} \cup \ldots$ $\cup a_{k}>b_{i} \cup a_{1} \cup \ldots \cup a_{k_{i}-1}$ and hence $a_{1} \cup \ldots \cup a_{k}=b_{i+1} \cup a_{1} \cup \ldots \cup a_{k_{i-1}}$ contrary to our assumption. Thus $b_{i} \cup a_{1} \cup \ldots \cup a_{k_{i}-1} \supset b_{i+1}$. If $b_{i} \cup a_{1} \cup \ldots$ $\cup a_{k_{i}-2} D b_{i+1}$, we have $b_{i} \cup a_{1} \cup \ldots \cup a_{k_{i}-1} \supset b_{i+1} \cup a_{1} \cup \ldots \cup a_{k_{i-2}} \supset b_{i} \cup a_{1}$ $\cup \ldots \cup a_{k_{i}-2}$. But $b_{i} \cup a_{1} \cup \ldots \cup a_{k_{i-1}}>b_{i} \cup a_{1} \cup \ldots \cup a_{k_{i-2}}$ and hence $b_{i} \cup a_{1} \cup \ldots \cup a_{k_{i-1}}=b_{i+1} \cup a_{1} \cup \ldots \cup a_{k_{i-2}}$ contrary to our assumption. Thus $b_{i} \cup a_{1} \cup \ldots \cup a_{k_{i}-2} \supset b_{i+1}$. Continuing in this manner we eventually have $b_{i} \cup a_{1} \supset b_{i+1}$. But then $b_{i} \cup a_{i} \supset b_{i+1} \supset b_{i}$ and $b_{i+1} \neq b_{i}$. Hence $b_{i+1}=b_{i} \cup a_{1}$ $=b_{i+1} \cup a_{1}$ which contradicts our assumption. We conclude, then, that at least two members of the above chain are equal. Thus (renumbering the $a$ 's if necessary) using the Birkhoff condition we have $a \subset b_{1} \subset \cdots \subset b_{i} \subset b_{i+1}<b_{i+1}$ $\cup a_{1}<\ldots<b_{i+1} \cup a_{1} \cup \ldots \cup a_{k_{i+1}-1}<a_{1} \cup \ldots \cup a_{k}$ where $k_{i+1} \leqq k_{i}-1 \leqq k$ $-(i+1)$. By induction, we get $a=b_{0} \subset b_{1} \subset \cdots \subset b_{r-1}<a_{1} \cup \cdots \cup a_{k}$ where $r \leqq k$. But then $b_{r}=a_{1} \cup \ldots \cup a_{k}$ and hence $r=l$ which contradicts $l>k$. Thus $l \leqq k$ and the lemma follows. 
The dual of condition $\mathrm{B} 1$ is the condition

B2. $a \cup b>b \rightarrow a>a \cap b$.

G. Birkhoff (Birkhoff [2]) has proved the following lemma which relates $\mathrm{B} 1$ and $\mathrm{B} 2$ to modularity.

Lemma 1.5. An archimedean lattice $\subseteq$ is modular if and only if B1 and B2 are satisfied.

2. Lattice ideals. A sublattice $\mathfrak{a}$ of $\subseteq$ is said to be an ideal if $x \supset a, a \in \mathfrak{a}$ implies $x \in \mathfrak{a}$. If $\mathfrak{a}$ consists of all elements $x$ such that $x \supset a$ for a fixed $a$, then $\mathfrak{a}$ is said to be a principal ideal and we write $\mathfrak{a}=(a)$. Now suppose that $\mathfrak{S}$ satisfies the descending chain condition. Then the set of elements in $\mathfrak{a}$ has a crosscut which can be expressed as a cross-cut of a finite number of them and hence belongs to $\mathfrak{a}$. Thus $\mathfrak{a}$ consists of all divisors of a fixed element of $\mathfrak{S}$ and hence is principal. Conversely, if every ideal of $\subseteq$ is principal, then a descending chain $a_{1} \supset a_{2} \supset \cdots$ generates an ideal $\mathfrak{a}$ which consists of all $x$ such that $x \supset a_{k}$ for some $k$. But then $\mathfrak{a}=(a)$ and $a \supset a_{k}$ for some $k$. Hence $a=a_{k}$ $=a_{k+1}=\cdots$ and every descending chain has only a finite number of distinct elements. We thus have

LEMma 2.1. $\subseteq$ satisfies the descending chain condition if and only if every ideal is principal.

The set of ideals of $\mathfrak{S}$ will be denoted by $\mathbb{R}$.

Definition 2.1. The union $\mathfrak{a} \cup \mathfrak{b}$ of two ideals $\mathfrak{a}$ and $\mathfrak{b}$ is the set of all elements $x$ such that $x \supset a \cup b$ for some $a \in \mathfrak{a}$ and $b \in \mathfrak{b}$. Similarly the cross-cut $\mathfrak{a} \cap \mathfrak{b}$ is the set of all elements $y$ such that $y \supset a \cap b$ for some $a \in \mathfrak{a}$ and $b \in \mathfrak{b}$.

It is readily verified that the union and cross-cut so defined are ideals and that $\mathfrak{R}$ is a lattice under these operations. The union $\mathfrak{a} \cup \mathfrak{b}$ is simply the settheoretic cross-cut of $\mathfrak{a}$ and $\mathfrak{b}$.

The definition of cross-cut may be readily extended to any subset $S$ of $\&$. $\Pi(S)$ consists of all elements of $\subseteq$ which belong to the cross-cut of a finite number of ideals of $S$. If $\subseteq$ has a unit element $u$, the union $\Sigma(S)$ is also defined and is simply the set-theoretic cross-cut of the ideals of $S$.

If $\mathfrak{a}$ and $\mathfrak{b}$ are principal ideals $\mathfrak{a}=(a)$ and $\mathfrak{b}=(b)$, then by Definition 2.1 $\mathfrak{a} \cup \mathfrak{b}=(a \cup b)$ and $\mathfrak{a} \cap \mathfrak{b}=(a \cap b)$. Hence the set of principal ideals forms a sublattice of $\mathfrak{R}$ which is isomorphic to $\subseteq$ and we may thus consider $\subseteq$ as a sublattice of $\mathbb{R}$.

LeMma 2.2. $\mathbb{R}$ is a modular (distributive) if and only if $\mathfrak{S}$ is modular (distributive).

Since $\mathfrak{S}$ is a sublattice of $\mathfrak{R}$, the modularity (distributivity) of $\mathbb{R}$ implies the modularity (distributivity) of $\subseteq$. 
Now let $\mathfrak{S}$ be distributive and let $x \in \mathfrak{a} \cup(\mathfrak{b} \cap \mathfrak{c})$. Then $x \supset a \cup(b \cap c)$ where $a \in \mathfrak{a}, b \in \mathfrak{b}$ and $c \in \mathfrak{c}$ by Definition 2.1. But then $x \supset a \cup(b \cap c) \supset(a \cup b) \cap(a \cup c)$ since $\mathfrak{S}$ is distributive and hence $x \in(\mathfrak{a} \cup \mathfrak{b}) \cap(\mathfrak{a} \cup \mathfrak{c})$. Thus $\mathfrak{a} \cup(\mathfrak{b} \cap \mathfrak{c}) \supset(\mathfrak{a} \cup \mathfrak{b})$ $\cap(\mathfrak{a} \cup \mathfrak{c})$. But $(\mathfrak{a} \cup \mathfrak{b}) \cap(\mathfrak{a} \cup \mathfrak{c}) \supset \mathfrak{a} \cup(\mathfrak{b} \cap \mathfrak{c})$ trivially. Hence $\mathfrak{l}$ is distributive. Now let $\subseteq$ be modular. Suppose $\mathfrak{a} \supset \mathfrak{b}$ and $x \in \mathfrak{b} \cup(\mathfrak{a} \cap \mathfrak{c})$. Then $x \supset b \cup(a \cap c)$ where $a \in \mathfrak{a}, b \in \mathfrak{b}$ and $c \in \mathfrak{c}$ by Definition 2.1. Now since $\mathfrak{a} \supset \mathfrak{b}$ we have $a \supset b_{1}$ where $b_{1} \in \mathfrak{b}$. But then $x \supset\left(b \cap b_{1}\right) \cup(a \cap c)$ and $a \supset b \cap b_{1}$ where $b_{1} \cap b \in \mathfrak{b}$. Hence $x \supset a \cap\left(\left(b \cap b_{1}\right) \cup c\right)$ since $\mathfrak{S}$ is modular and $x \in \mathfrak{a} \cap(\mathfrak{b} \cup \mathfrak{c})$. Thus $\mathfrak{b} \cup(\mathfrak{a} \cap \mathfrak{c})$ $\supset \mathfrak{a} \cap(\mathfrak{b} \cup \mathfrak{c})$ and since $\mathfrak{a} \cap(\mathfrak{b} \cup \mathfrak{c}) \supset \mathfrak{b} \cup(\mathfrak{a} \cap \mathfrak{c})$ trivially, $\mathfrak{R}$ is modular. This completes the proof.

LEMMA 2.3. Let $\mathfrak{a} \supset \mathfrak{b} \supset \ldots \supset \mathfrak{u} \supset \ldots$ be a chain of ideals such that $\mathfrak{u} \supset(a)$ and $\mathfrak{u} \neq(a)$ for all ideals of the chain. Then if $\mathfrak{p}$ is the cross-cut of the ideals of the chain, $\mathfrak{p} \supset($ a $)$ and $\mathfrak{p} \neq(a)$.

We note that $\mathfrak{p}$ is the set-theoretic union of the elements of the ideals $\mathfrak{a}, \mathfrak{b}, \cdots, \mathfrak{u}, \cdots$. For if $x \in \mathfrak{p}$, then $x$ divides a finite cross-cut of the ideals of the chain and hence divides some ideal of the chain. Now suppose $p=a$. Then $a \in \mathfrak{p}$ and $a \in \mathfrak{u}$ for some $\mathfrak{u}$. But then $\mathfrak{u}=(a)$ contrary to assumption. Hence $p \neq a$.

The results so far have been independent of the well ordering hypothesis. However, to prove the fundamental property of the ideals we must assume that the elements of $\subseteq$ can be well ordered. This will be assumed through the remainder of the paper.

TheOREM 2.1. Let $\mathfrak{b} \supset(a)$ and $\mathfrak{b} \neq(a)$. Then there exists an ideal $\mathfrak{p}$ such that $\mathfrak{b} \supset \mathfrak{p}>(a)$.

Proof. Let $U$ be the set of all elements $x$ such that $x \supset a$. Let $U$ be well ordered, $U=\left\{x_{\nu}\right\}, \nu<\sigma$. Define $\mathfrak{a}_{0}=\mathfrak{b}$. Now suppose that $\mathfrak{a}_{\mu}$ has been defined for all $\mu<\nu$ in such a way that $\mathfrak{a}_{\mu} \neq(a), \mathfrak{a}_{\mu} \supset \mathfrak{a}_{\mu^{\prime}}$ if $\mu \leqq \mu^{\prime}$, and $\mathfrak{a}_{\mu} \cap x_{\mu}=\mathfrak{a}_{\mu}$ or $\mathfrak{a}_{\mu} \cap x_{\mu}=a$. Let $\mathfrak{c}_{\nu}$ be the cross-cut of all $\mathfrak{a}_{\mu}$ with $\mu<\nu$. Then $\mathfrak{c}_{\nu} \neq(a)$ by Lemma 2.3. If $\mathfrak{c}_{\nu} \cap x_{\nu} \neq(a)$, let $\mathfrak{a}_{\nu}=\mathfrak{c}_{\nu} \cap x_{\nu}$; otherwise let $\mathfrak{a}_{\nu}=\mathfrak{c}_{\nu}$. Then $\mathfrak{a}_{\nu} \neq(a)$ and $\mathfrak{a}_{\mu} \supset \mathfrak{a}_{\nu}$, all $\mu<\nu$. Clearly $\mathfrak{a}_{\nu} \cap x_{\nu}=a$ or $\mathfrak{a}_{\nu}$. Now let $\mathfrak{p}=\Pi_{\nu<\sigma} \mathfrak{a}_{\nu}$. Then $\mathfrak{p} \neq a$ by Lemma 2.3 and $\mathfrak{b} \supset \mathfrak{p}$. If $\mathfrak{p} \supset \mathfrak{a} \supset(a)$ and $\mathfrak{p} \neq \mathfrak{a}$, there exists an element $x \in \mathfrak{a}$ such that $x \notin \mathfrak{p}$. Since $x \supset a$ we have $x=x_{\nu}$ for some $\nu$. But then $\mathfrak{a}_{\nu} \cap x=a$ since otherwise $x_{\nu} \supset \mathfrak{a}_{\nu} \supset \mathfrak{p}$ which contradicts $x \notin \mathfrak{p}$. Thus $a=\mathfrak{a}_{\nu} \cap x \supset \mathfrak{p} \cap \mathfrak{a}=\mathfrak{a} \supset a$ and $\mathfrak{a}=(a)$. Hence $\mathfrak{p}>a$.

In the special instances of Boolean algebras and distributive lattices, Theorem 2.1 gives respectively the existence of the prime ideals of Stone (Stone [1]) and the maximal collections of Wallman (Wallman [1]).

We next prove a theorem which enables us to pass from ideal relations to the corresponding element relations. The following lemma is required.

Lemma 2.4. Let $\mathfrak{a}=\mathfrak{a}\left(\mathfrak{a}_{1}, \cdots, \mathfrak{a}_{n}\right)$ be an ideal obtained from the ideals 
$\mathfrak{a}_{1}, \cdots, \mathfrak{a}_{n}$ by forming a finite number of unions and cross-cuts. Then if $x \in \mathfrak{a}$, there exist elements $a_{1}, \cdots, a_{n}, a_{i} \in \mathfrak{a}_{i}$, such that $x \supset \mathfrak{a}\left(a_{1}, \cdots, a_{n}\right)$.

For let $n(\mathfrak{a})$ denote the number of union and cross-cut symbols in the expression $\mathfrak{a}\left(\mathfrak{a}_{1}, \cdots, \mathfrak{a}_{n}\right)$. Suppose that the lemma is true for all expressions $\mathfrak{a}$ for which $n(\mathfrak{a})<k$. Let $n(\mathfrak{a})=k$. Then $\mathfrak{a}=a_{1} \circ a_{2}$ where $\circ$ is either $\cap$ or $\cup$ and $n\left(\mathfrak{a}_{1}\right)<k, n\left(\mathfrak{a}_{2}\right)<k$. Now if $x \in \mathfrak{a}$ we have $x \supset x_{1} \circ x_{2}$ where $x_{1} \in \mathfrak{a}_{1}$ and $x_{2} \in \mathfrak{a}_{2}$ by the definition of union and cross-cut. But then by the induction assumption elements $a_{1}^{\prime}, \cdots, a_{n}^{\prime}$ and $a_{1}^{\prime \prime}, \cdots, a_{n}^{\prime \prime}$ exist such that $x_{1} \supset a_{1}\left(a_{1}^{\prime}, \cdots, a_{n}^{\prime}\right)$, $x_{2} \supset \mathfrak{a}_{2}\left(a_{1}^{\prime}, \cdots, a_{n}^{\prime \prime}\right)$. Let $a_{i}=a_{i}^{\prime} \cap a_{i}^{\prime \prime}$. Then $x \supset x_{1} \circ x_{2} \supset \mathfrak{a}_{1}\left(a_{1}^{\prime}, \cdots, a_{n}^{\prime}\right)$ $\circ \mathfrak{a}_{2}\left(a_{1}^{\prime}, \cdots, a_{n}^{\prime \prime}\right) \supset \mathfrak{a}_{1}\left(a_{1}, \cdots, a_{n}\right) \circ \mathfrak{a}_{2}\left(a_{1}, \cdots, a_{n}\right)=\mathfrak{a}\left(a_{1}, \cdots, a_{n}\right)$ and $a_{i}$ is clearly in $\mathfrak{a}_{i}$. Since the lemma is trivially true when $n(\mathfrak{a})=1$ by Definition 2.1, the proof is complete.

THEOREM 2.2. Let $(a)=\mathfrak{a}\left(\mathfrak{a}_{1}, \cdots, \mathfrak{a}_{n}\right)$ where $\mathfrak{a}$ is obtained from $\mathfrak{a}_{1}, \cdots, \mathfrak{a}_{n}$ by forming a finite number of union and cross-cuts. Then $(a)=\mathfrak{a}\left(a_{1}, \cdots, a_{n}\right)$ where $a_{i} \in \mathfrak{a}_{i}$.

Proof. By Lemma $2.4 a \supset \mathfrak{a}\left(a_{1}, \cdots, a_{n}\right)$ where $a_{i} \in \mathfrak{a}_{i}$. But then $\mathfrak{a}\left(a_{1}, \cdots, a_{n}\right) \supset \mathfrak{a}\left(\mathfrak{a}_{1}, \cdots, \mathfrak{a}_{n}\right)=(a)$. Hence $(a)=\mathfrak{a}\left(a_{1}, \cdots, a_{n}\right)$.

As an example, if $a=\mathfrak{a}_{1} \cap \cdots \cap \mathfrak{a}_{n}$ then elements $a_{i} \in \mathfrak{a}_{i}$ exist such that $a=a_{1} \cap \cdots \cap a_{n}$.

We conclude this section with two useful lemmas on irreducibles $\left({ }^{7}\right)$.

Lемма 2.5. If $q$ is irreducible in $\mathfrak{S}$, then $q$ is irreducible in $\mathbb{R}$.

For if $q$ is reducible in $\mathfrak{R}$, then $q=\mathfrak{a} \cap \mathfrak{b}, \mathfrak{a}, \mathfrak{b} \neq q$. But then $q=a \cap b, a \in \mathfrak{a}$, $b \in \mathfrak{b}$ by Theorem 2.2. Clearly $a \neq q$ and $b \neq q$. Hence $q$ is reducible in $\mathfrak{\Im}$. Inverting the logic gives the lemma.

LEMMA 2.6. Let every element of $\subseteq$ be expressible as a cross-cut of irreducibles. Then if $\mathfrak{a} \supset \mathfrak{b}, \mathfrak{a} \neq \mathfrak{b}$, there exists an irreducible $q$ of $\mathfrak{S}$ such that $q \supset \mathfrak{b}, q \supset \mathfrak{a}$.

For since $\mathfrak{a} \neq \mathfrak{b}, b$ exists such that $b \in \mathfrak{b}, b \in \mathfrak{a}$. Let $b=q_{1} \cap \cdots \cap q_{k}$. If $q_{i} \in \mathfrak{a}$ for every $i$ then $b \in \mathfrak{a}$ contrary to assumption. Hence $q_{i} \notin \mathfrak{a}$ for some $i$. But then $q_{i} \supset b \supset \mathfrak{b}$.

3. Birkhoff lattices. In D1 and D2 a lattice satisfying B1 was defined to be a Birkhoff lattice. Since both the ascending and descending chain conditions were assumed to hold, B1 was never satisfied trivially. Now in a sufficiently general lattice no covering relations may exist and B1 will hold vacuously. Hence we formulate a more general definition which reduces to that used in D1 and D2 if the descending chain condition holds.

Definition 3.1. A lattice $\subseteq$ is said to be a Birkhoff lattice if each element of $\mathbb{S}$ satisfies the Birkhoff condition in the lattice of ideals.

(7) An element $q$ is said to be cross-cut irreducible or simply irreducible if $q=a \bigcap b \rightarrow q=a$ or $q=b . q$ is said to be union irreducible if $q=a \cup b \rightarrow q=a$ or $q=b$. 
A lattice $\subseteq$ is never vacuously a Birkhoff lattice since by Theorem 2.1 covering ideals always exist. Furthermore if the descending chain condition holds, then every ideal is principal and $\subseteq$ is a Birkhoff lattice if and only if B1 holds in $\subseteq$.

Now if $\mathfrak{S}$ has a unit element $u$ and $a$ is any element of $\mathfrak{S}$, then the union of the ideals covering $a$ exists and will be denoted by $\mathfrak{u}_{a}$. Let $\mathfrak{R}_{a}$ denote the quotient lattice of all ideals of $\mathbb{R}$ which are divisible by $\mathfrak{u}_{a}$ and which divide $a$. Then $\mathfrak{R}_{a}$ is a dense sublattice of $\mathfrak{R}$ and every proper divisor of $a$ in $\mathfrak{R}$ divides some point ideal of $\mathfrak{R}_{a}$ by Theorem 2.1 . Clearly $\mathfrak{R}_{a}$ reduces to the sublattice $\mathfrak{S}_{a}$ of the previous papers if the descending chain condition holds. The essential properties of $\mathfrak{S}_{a}$ followed from its finite dimensionality. But $\mathfrak{R}_{a}$ is in general not finite dimensional. However we now prove a theorem which insures the archimedean character of $\mathfrak{R}_{a}$ in most cases of arithmetical interest. We need the following lemma:

Lemмa 3.1. Let $\subseteq$ be a Birkhoff lattice. Then if $\mathfrak{p}_{1}, \cdots, \mathfrak{p}_{k}$ is a maximal independent set of point ideals of $\mathfrak{R}_{a}$, the length of any chain of $\mathfrak{R}_{a}$ is not greater than $k$.

Since the length of any chain is one less than the number of distinct members of the chain, the lemma follows immediately from Lemma 1.4 and Definition 3.1 .

According to Lemma $3.1, \mathfrak{R}_{a}$ is archimedean if and only if $\mathfrak{u}_{a}$ can be expressed as a union of a finite number of point ideals of $\mathfrak{R}_{a}$.

THEOREM 3.1. Let $\subseteq$ be a Birkhoff lattice in which every element may be represented as a cross-cut of irreducibles. Then $\mathfrak{R}_{a}$ is archimedean if and only if the number of components in the irreducible decompositions of $a$ is bounded.

Proof. Let the number of components in the irreducible decompositions of $a$ be bounded, say less than $n$. Then if $\mathfrak{R}_{a}$ is not archimedean, by Lemmas 1.2 and 3.1 there are $n$ union independent point ideals $\mathfrak{p}_{1}, \cdots, \mathfrak{p}_{n}$ of $\mathfrak{R}_{a}$ which generate a Boolean algebra. Let $\mathfrak{a}_{i}=\mathfrak{p}_{1} \cup \ldots \cup_{\mathfrak{p}_{i-1}} \cup \mathfrak{p}_{i+1} \cup \ldots \cup_{\mathfrak{p}_{n}}$. Then $a=\mathfrak{a}_{1} \cap \cdots \cap \mathfrak{a}_{n}$. Hence by Theorem $2.2 a=a_{1} \cap \cdots \cap a_{n}$ where $a_{i} \in \mathfrak{a}_{i}$. Now let $a_{i}=q_{i 1} \cap \cdots \cap q_{i k_{i}}$ where $q_{i 1}, \cdots, q_{i k_{i}}$ are irreducibles of $\mathfrak{S}$. Then $a=q_{11} \cap q_{12} \cap \cdots \cap q_{n k_{n}}$ and this representation may be reduced $\left({ }^{8}\right)$ by dropping our superfluous irreducibles. However not all of the irreducibles belonging to any one $a_{i}$ may be dropped out since otherwise $a=q_{11} \cap \cdots \cap q_{n k_{n}}$ $\supset a_{1} \cap \cdots \cap a_{i-1} \cap a_{i+1} \cap \cdots \cap a_{n} \supset \mathfrak{a}_{1} \cap \ldots \cap \mathfrak{a}_{i-1} \cap \mathfrak{a}_{i+1} \cap \cdots \cap \mathfrak{a}_{n} \supset \mathfrak{p}_{i}$ contrary to $\mathfrak{p}_{i}>a$. Hence $a$ has a decomposition having at least $n$ components. But this contradicts our assumption that the number of components is less than $n$. Hence $\mathfrak{R}_{a}$ is archimedean and of length less than $n$.

On the other hand let the number of components be unbounded. Then for

( $\left.{ }^{8}\right)$ A representation $a=a_{1} \cap a_{2} \cap \cdots \cap a_{n}$ is said to be reduced if $a_{1}, \cdots, a_{n}$ are cross-cut independent. 
every $k$ there is an irreducible decomposition $a=q_{1} \cap \cdots \cap q_{n}$ with $n \geqq k$. Let $q_{i}^{\prime}=q_{1} \cap \cdots \cap q_{i-1} \cap q_{i+1} \cap \cdots \cap q_{n}$. Then $q_{i}^{\prime} \supset a$ and $q_{i}^{\prime} \neq a$ since the representation is reduced. Hence $q_{i}^{\prime} \supset p_{i}>a$ by Theorem 2.1. Suppose $\mathfrak{p}_{1} \cup \ldots \cup \mathfrak{p}_{i-1} \cup \mathfrak{p}_{i+1} \cup \ldots \cup \mathfrak{p}_{n} \supset \mathfrak{p}_{i}$. Then $q_{i} \supset q_{1}^{\prime} \cup \ldots \cup q_{q_{i-1}^{\prime}} \cup q_{q_{i+1}}^{\prime} \cup \ldots$ $\cup q_{n}^{\prime} \supset \mathfrak{p}_{1} \cup \ldots \cup \cup_{\mathfrak{p}_{i-1}} \cup \mathfrak{p}_{i+1} \cup \ldots \cup \cup_{\mathfrak{p}_{n}} \supset \mathfrak{p}_{i}$ and $a=q_{i} \cap q_{i}^{\prime} \supset \mathfrak{p}_{i}$ which contradicts $\mathfrak{p}_{i}>a$. Thus $\mathfrak{p}_{1}, \cdots, \mathfrak{p}_{n}$ are union independent. Hence for every $k$ there are more than $k$ union independent point ideals of $\mathfrak{R}_{a}$ and $\mathfrak{R}_{a}$ is not archimedean.

If $\mathfrak{R}_{a}$ is archimedean it has some simple structure properties which follow from the Birkhoff condition.

THEOREM 3.2. Let $\mathfrak{S}$ be a Birkhoff lattice. Then if $\mathfrak{R}_{a}$ is archimedean, it is complemented and every ideal can be expressed as a cross-cut of simple ideals.

Proof. Let $\mathfrak{a} \in \mathfrak{R}_{a}$ and let $\mathfrak{p}_{1}, \cdots, \mathfrak{p}_{k}$ be a maximal independent set of point ideals of $\mathfrak{R}_{a}$ divisible by $\mathfrak{a}$. Imbed $\mathfrak{p}_{1}, \cdots, \mathfrak{p}_{k}$ in a maximal independent set $\mathfrak{p}_{1}, \cdots, \mathfrak{p}_{n}$. Let $\mathfrak{a}^{\prime}=\mathfrak{p}_{k+1} \cup \ldots \cup \mathfrak{p}_{n}$. Then $\mathfrak{a} \cup \mathfrak{a}^{\prime} \supset \mathfrak{p}_{1} \cup \ldots \cup \mathfrak{p}_{n} \supset \mathfrak{u}_{a}$. Hence $\mathfrak{a} \cup \mathfrak{a}^{\prime}=\mathfrak{u}_{a}$. Now suppose that $\mathfrak{a} \cap \mathfrak{a}^{\prime} \neq a$. Then $\mathfrak{a} \cap \mathfrak{a}^{\prime} \supset \mathfrak{p}>a$ by Theorem 2.1 . Since $\mathfrak{a} \supset \mathfrak{p}$ we have $\mathfrak{p}_{1} \cup \ldots \cup \mathfrak{p}_{k} \supset \mathfrak{p}$ by the maximal property of $\mathfrak{p}_{1}, \cdots, \mathfrak{p}_{k}$ and $a=\left(\mathfrak{p}_{1} \cup \ldots \cup \mathfrak{p}_{k}\right) \cap \mathfrak{a}^{\prime} \supset \mathfrak{p}$, which contradicts $\mathfrak{p}>a$. Hence $\mathfrak{a} \cap \mathfrak{a}^{\prime}=a$ and $\mathfrak{R}_{a}$ is complemented.

Now let $q$ be irreducible in $\mathfrak{R}_{a}$. Let $\mathfrak{p}_{1}, \cdots, \mathfrak{p}_{k}$ be a maximal independent set of point ideals of $\mathfrak{R}_{a}$ divisible by $\mathfrak{q}$ and let this set be imbedded in a maximal independent set $\mathfrak{p}_{1}, \cdots, \mathfrak{p}_{k}, \cdots, \mathfrak{p}_{n}$. Then $\mathfrak{q} D \mathfrak{p}_{k+1}, \cdots, \mathfrak{p}_{n}$ and hence $\mathfrak{q} \cup \mathfrak{p}_{i}>\mathfrak{q}, i=k+1, \cdots, n$, by $\mathrm{B} 1^{\prime}$. But since $\mathfrak{q}$ is irreducible in $\mathfrak{R}_{a}$ we have $\mathfrak{q} \cup_{\mathfrak{p}_{k+1}}=\cdots=\mathfrak{q} \cup_{\mathfrak{p}_{n}}$. Hence $\mathfrak{u}_{a}=\mathfrak{q} \cup \cup_{\mathfrak{u}_{a}}=\mathfrak{q} \cup \mathfrak{p}_{k+1} \cup \ldots \cup \mathfrak{q} \cup_{\mathfrak{p}_{n}}=\mathfrak{q} \cup_{\mathfrak{p}_{k+1}}$ $>q$. Thus each ideal which is irreducible in $\mathfrak{R}_{a}$ is a simple ideal of $\mathfrak{R}_{a}$ and since $\mathfrak{R}_{a}$ is archimedean each ideal of $\mathfrak{R}_{a}$ can be represented as a cross-cut of simple ideals.

If $\mathfrak{R}_{a}$ is not archimedean it will in general neither be complemented nor will every ideal be expressible as a cross-cut of simple ideals $\left({ }^{9}\right)$. In the archimedean case an arbitrary complement of $\mathfrak{a}$ in $\mathfrak{R}_{a}$ will be denoted by $\mathfrak{a}^{\prime}$.

Definition 3.2. An ideal $\mathfrak{c} \neq \mathfrak{u}_{a}$ of $\mathfrak{R}_{a}$ is said to be characteristic if there exists an irreducible $q$ of $\subseteq$ which divides exactly the same point ideals of $\mathfrak{R}_{a}$ as $c$.

THEOREM 3.3. An element $a \in \subseteq$ has a reduced representation $a=q_{1} \cap \cdots \cap q_{n}$ where $q_{1}, \cdots, q_{n}$ are irreducibles if and only if a has a reduced representation $a=\mathfrak{c}_{1} \cap \cdots \cap \mathfrak{c}_{n}$ where $\mathfrak{c}_{1}, \cdots, \mathfrak{c}_{n}$ are characteristic ideals of $\mathfrak{R}_{a}$ such that $q_{i} \supset \mathfrak{c}_{i}$.

Proof. Let $a=q_{1} \cap \cdots \cap q_{n}$ be a reduced representation of $a$ as a cross-cut of irreducibles. If $q_{i} \supset \mathfrak{u}_{a}$ for some $i$, then $q_{1} \cap \cdots \cap q_{i-1} \cap q_{i+1} \cap \cdots \cap q_{n} \supset \mathfrak{p}_{i}$ $>a$ and hence $a=q_{1} \cap \cdots \cap q_{n} \supset \mathfrak{p}_{i}>a$, which is impossible. Thus $q_{i} D \mathfrak{u}_{a}$. Let $\mathfrak{c}_{i}$ be a characteristic ideal associated with $q_{i}$. There is always at least one

(9) See $\$ 7$ for an example. 
such ideal, namely, the union of the point ideals of $\mathfrak{R}_{a}$ divisible by $q_{i}$. Now $a=q_{1} \cap \cdots \cap q_{n} \supset \mathfrak{c}_{1} \cap \cdots \cap \mathfrak{c}_{n} \supset a$ implies $a=\mathfrak{c}_{1} \cap \cdots \cap \mathfrak{c}_{n}$. Suppose $\mathfrak{c}_{i} \supset \mathfrak{c}_{1} \cap \cdots \cap \mathfrak{c}_{i-1} \cap \mathfrak{c}_{i+1} \cap \cdots \cap \mathfrak{c}_{n}$. Then $q_{i} \cap \cdots \cap q_{i-1} \cap q_{i+1} \cap \cdots \cap q_{n}$ $\supset \mathfrak{p}_{i}>a$ implies $\mathfrak{c}_{1} \cap \cdots \cap \mathfrak{c}_{i-1} \cap \mathfrak{c}_{i+1} \cap \cdots \cap \mathfrak{c}_{n} \supset \mathfrak{p}_{i}$. But then $a=\mathfrak{c}_{i} \cap \mathfrak{c}_{1}$ $\cap \cdots \cap \mathfrak{c}_{i-1} \cap \mathfrak{c}_{i+1} \cap \cdots \cap \mathfrak{c}_{n} \supset \mathfrak{p}_{i}$ which is impossible. Hence the representation $a=\mathfrak{c}_{1} \cap \cdots \cap \mathfrak{c}_{n}$ is reduced.

Now let $a=\mathfrak{c}_{1} \cap \cdots \cap \mathfrak{c}_{n}$ where $\mathfrak{c}_{1}, \cdots, \mathfrak{c}_{n}$ are characteristic ideals and the representation is reduced. Let $q_{1}, \cdots, q_{n}$ be associated irreducibles. Suppose $q_{1} \cap \cdots \cap q_{n} \supset \mathfrak{p}>a$. Then $a=\mathfrak{c}_{1} \cap \cdots \cap \mathfrak{c}_{n} \supset \mathfrak{p}>a$ which is impossible. Hence $a=q_{1} \cap \cdots \cap q_{n}$. It follows easily that this representation is reduced.

The characteristic ideals of $\mathfrak{R}_{a}$ can be characterized in terms of the structure of $\mathfrak{R}$ as follows:

Theorem 3.4. Let $\subseteq$ be a Birkhoff lattice in which each element can be expressed as a cross-cut of irreducibles. Then if $\mathfrak{R}_{a}$ is archimedean, $\mathrm{c}$ is characteristic if and only if there exists an ideal $\mathfrak{x} \in \mathbb{R}$ such that $\mathfrak{x} \supset \mathfrak{c}, \mathfrak{c}^{\prime} \cup \mathfrak{x}>\mathfrak{x}$ and $\mathfrak{c}^{\prime} \cap \mathfrak{x}=a$ for every $\mathrm{c}^{\prime}$.

Proof. Let us first assume that such an ideal $\mathfrak{x}$ exists. Then $\mathfrak{u}_{a} \cup \mathfrak{x}=\mathfrak{c} \cup \mathfrak{c}^{\prime} \cup \mathfrak{x}$ $=\mathfrak{c}^{\prime} \cup \mathfrak{x}$. Let $q$ be an irreducible such that $q \supset \mathfrak{x}, q \rrbracket \mathfrak{u}_{a} \cup \mathfrak{x}$ (Lemma 2.6). Since $q \supset \mathfrak{x} \supset \mathfrak{c}, q$ divides every point ideal of $\mathfrak{R}_{a}$ which $\mathfrak{c}$ divides. Now let $q \supset \mathfrak{p}$. Then if $\mathfrak{x} D \mathfrak{p}$ we have $\mathfrak{c}^{\prime} \cup \mathfrak{x}=\mathfrak{u}_{a} \cup \mathfrak{x} \supset \mathfrak{p} \cup \mathfrak{x} \supset \mathfrak{x}$ and $\mathfrak{p} \cup \mathfrak{x} \neq \mathfrak{x}$. Hence $\mathfrak{c}^{\prime} \cup \mathfrak{x}=\mathfrak{p} \cup \mathfrak{x}$ and $q \supset \mathfrak{p} \cup \mathfrak{x} \supset \mathfrak{c}^{\prime} \cup \mathfrak{x}$ which contradicts the definition of $q$. Hence $\mathfrak{x} \supset \mathfrak{p}$. Now if $\mathfrak{c} D \mathfrak{p}$, then $\mathfrak{c}^{\prime} \supset \mathfrak{p}$ for some $\mathfrak{c}^{\prime}$. But then $a=\mathfrak{c}^{\prime} \cap \mathfrak{x} \supset \mathfrak{p}$ which is impossible. Hence $q \supset \mathfrak{p}$ implies $c \supset p$ and $c$ is thus characteristic.

On the other hand let $c$ be characteristic and let $q$ be an irreducible associated with $c$. Then $q \cup \mathfrak{c}^{\prime}>q$ for every $\mathfrak{c}^{\prime}$. For there is a point ideal $\mathfrak{p}$ such that $\mathfrak{c}^{\prime} \supset \mathfrak{p}, \mathfrak{c} D \mathfrak{p}$ since otherwise we would have $\mathfrak{c}^{\prime}=a$ and $\mathfrak{c}=\mathfrak{u}_{a}$ contrary to the definition of a characteristic ideal. Now $q \cup_{\mathfrak{p}}=q \cup_{\mathfrak{u}_{a}}>q$ since $q$ is irreducible in $\mathfrak{R}$ by Lemma 2.5. Hence $q \cup \mathfrak{u}_{a}=q \cup \mathfrak{c}^{\prime}=q \cup \mathfrak{p}>q$. Now if $\mathfrak{c}^{\prime} \cap q \neq a$, then $\mathfrak{c}^{\prime} \cap q \supset \mathfrak{p}>a$ and hence $\mathfrak{c}^{\prime} \supset \mathfrak{p}, q \supset \mathfrak{p}$ by Theorem 2.2. But then $\mathfrak{c} \supset \mathfrak{p}$ and hence $a=\mathfrak{c} \cap \mathfrak{c}^{\prime} \supset \mathfrak{p}$ which is impossible. Thus $\mathfrak{c}^{\prime} \cap q=a$ for every $\mathfrak{c}^{\prime}$.

COROLlARY 3.1. Each simple ideal of $\mathfrak{R}_{a}$ is characteristic.

We may take $\mathfrak{x}$ to be the simple ideal itself.

TheOREM 3.5. Let $\mathfrak{\subseteq}$ be a Birkhoff lattice in which each element can be expressed as a cross-cut of irreducibles. Then if $\mathfrak{R}_{a}$ is archimedean, each characteristic ideal $\mathfrak{c}$ of $\mathfrak{R}_{a}$ occurs in a reduced representation $a=\mathfrak{c} \cap \mathfrak{c}_{1} \cap \ldots \cap \mathfrak{c}_{k}$ where $k$ is the number of maximal independent point ideals divisible by $c$ and $\mathfrak{c}_{1}, \cdots, \mathfrak{c}_{k}$ are characteristic ideals of $\mathfrak{R}_{a}$.

Proof. Let $\mathfrak{p}_{1}, \cdots, \mathfrak{p}_{k}$ be a maximal independent set of point ideals of $\mathfrak{R}_{a}$ divisible by $c$. Imbed $\mathfrak{p}_{1}, \cdots, \mathfrak{p}_{k}$ in a maximal independent $\operatorname{set} \mathfrak{p}_{1}, \cdots, \mathfrak{p}_{k}, \cdots, \mathfrak{p}_{n}$. Let $\mathfrak{c}_{i}=\mathfrak{p}_{1} \cup \ldots \cup \cup_{\mathfrak{p}_{i-1}} \cup \mathfrak{p}_{i+1} \cup \ldots \cup \mathfrak{p}_{k} \cup \ldots \cup \mathfrak{p}_{n}, i=1, \cdots, k$. If $\mathfrak{c} \cap \mathfrak{c}_{1}$ 
$\cap \ldots \cap \mathfrak{c}_{k} \neq a$ we have $\mathfrak{c} \cap \mathfrak{c}_{1} \cap \ldots \cap \mathfrak{c}_{k} \supset \mathfrak{p}>a$ and $\mathfrak{c} \supset \mathfrak{p}$ implies $\mathfrak{p}_{1} \cup \ldots$ $\cup \mathfrak{p}_{k} \supset \mathfrak{p}$. But then $a=\left(\mathfrak{p}_{1} \cup \ldots \cup \mathfrak{p}_{k}\right) \cap \mathfrak{c}_{1} \cap \ldots \cap \mathfrak{c}_{k} \supset \mathfrak{p}$ which is impossible. Hence $a=\mathfrak{c} \cap \mathfrak{c}_{1} \cap \cdots \cap \mathfrak{c}_{k}$. Also since $\mathfrak{c} \cap \mathfrak{c}_{1} \cap \cdots \cap \mathfrak{c}_{i-1} \cap \mathfrak{c}_{i+1} \cap \cdots \cap \mathfrak{c}_{k}$ $\supset \mathfrak{p}_{i}$ the representation is reduced. Since $\mathfrak{c}_{1}, \cdots, \mathfrak{c}_{k}$ are simple ideals of $\mathfrak{l}_{a}$, they are characteristic by Corollary 3.1 .

COROLlaRy 3.2. Let $\subseteq$ be a Birkhoff lattice in which every element can be expressed as a cross-cut of irreducibles. Then if $\mathfrak{L}_{a}$ is archimedean of length $k$, $a$ has a reduced decomposition into irreducibles with $k$ components.

For by Lemma 1.2 and Theorem 3.4, $a$ has a reduced representation as a cross-cut of $k$ characteristic ideals of $\mathfrak{R}_{a}$.

Lemma 3.2. Let $\subseteq$ be a Birkhoff lattice and let $\mathfrak{R}_{a}$ be archimedean for some a. Then $\mathfrak{R}_{a}$ is modular if and only if it satisfies B2.

For let $\mathfrak{R}_{a}$ satisfy B2 and let $q$ be a union irreducible ideal of $\mathfrak{R}_{a}$. If $\mathbb{\nabla} \mathbb{q}$ and $z$ is a simple ideal of $\mathfrak{R}_{a}$ we have $q>\mathfrak{q} \cap \mathbb{z}$ by B2. Hence since $q$ is union irreducible we have $\mathfrak{q} \cap \mathfrak{z}=\mathfrak{q} \cap \mathfrak{z}^{\prime}$ for any two simple ideals $z$ and $\mathfrak{z}^{\prime}$ which do not divide $\mathfrak{q}$. Let $a=\mathfrak{z}_{1} \cap \cdots \cap \mathfrak{z}_{n}$ where $\mathfrak{z}_{1}, \cdots, \mathbb{z}_{l} \supset \mathfrak{q} ; \mathfrak{z}_{l+1}, \cdots, \mathfrak{z}_{n} D \mathfrak{q}$. Then $a=\mathfrak{q} \cap a=\mathfrak{q} \cap z_{1} \cap \cdots \cap z_{n}=\left(\mathfrak{q} \cap \mathfrak{z}_{l+1}\right) \cap \cdots \cap\left(\mathfrak{q} \cap z_{n}\right)=\mathfrak{q} \cap \mathfrak{z}_{l+1}<\mathfrak{q}$. Hence $\mathfrak{q}$ is a point of $\mathfrak{R}_{a}$ and every ideal of $\mathfrak{R}_{a}$ is a union of point ideals. Now let $\mathfrak{a}>\mathfrak{a} \cap \mathfrak{b}$ in $\mathfrak{R}_{a}$. Then since every ideal is a union of point ideals, there exists a point ideal $\mathfrak{p}$ such that $\mathfrak{a} \supset \mathfrak{p}, \mathfrak{a} \cap \mathfrak{b} D \mathfrak{p}$. But then $\mathfrak{a}=(\mathfrak{a} \cap \mathfrak{b}) \cup \mathfrak{p}$. Hence $\mathfrak{a} \cup \mathfrak{b}=(\mathfrak{a} \cap \mathfrak{b}) \cup \mathfrak{p} \cup \mathfrak{b}$ $=\mathfrak{p} \cup \mathfrak{b}>\mathfrak{b}$ since $\mathfrak{S}$ is a Birkhoff lattice. Thus B1 and B2 hold in $\mathfrak{R}_{a}$ and $\mathfrak{R}_{a}$ is modular by Lemma 1.5. Conversely, if $\mathfrak{R}_{a}$ is modular, then B2 is satisfied by Lemma 1.5. This completes the proof.

According to Theorem 3.1, if every element of a lattice $\mathfrak{S}$ has a decomposition into irreducibles and the number of components in the decompositions of $a$ is bounded, then $\mathfrak{R}_{a}$ is archimedean. This result can be sharpened considerably if $\subseteq$ is modular.

Lemma 3.3. Let $\subseteq$ be a modular lattice. Then if an element a has a decomposition into irreducibles, $\mathfrak{R}_{a}$ is archimedean.

For let $a=q_{1} \cap \cdots \cap q_{k}$ where $q_{1}, \cdots, q_{k}$ are irreducible. Since $\subseteq$ is modular, $\mathfrak{R}$ is modular by Lemma 2.2. Now if $q_{i} D \mathfrak{p}$ where $\mathfrak{p}>a$, we have $q_{i} \cup \mathfrak{p}>q_{i}$ and hence $q_{i} \cup \mathfrak{u}_{a}>q_{i}$ since $q_{i}$ is irreducible. But then $\mathfrak{u}_{a}>\mathfrak{u}_{a} \cap q_{i}$ since $\mathfrak{R}$ is modular. Thus each irreducible $q_{i}$ divides a simple characteristic ideal $\mathfrak{c}_{i}=q_{i} \cap \mathfrak{u}_{a}$. Since $\mathfrak{R}$ is modular, we have $\mathfrak{u}_{a}>\mathfrak{c}_{1}>\mathfrak{c}_{1} \cap \mathfrak{c}_{2}>\cdots>\mathfrak{c}_{1} \cap \cdots \cap \mathfrak{c}_{k}$ $=a$. Hence $\mathfrak{R}_{a}$ is archimedean and the lemma is proved.

If $\subseteq$ is modular and $a$ has two reduced decompositions into irreducibles, then by Lemma 3.3, $\mathfrak{R}_{a}$ is archimedean and $a$ has two reduced representations as a cross-cut of simple ideals. Now by Lemma 3.2, B2 holds in $\mathfrak{R}_{a}$ and hence by the dual of Lemma 1.3 any two reduced representations of $a$ as a cross-cut of simple ideals have the same number of components and any simple ideal 
of one decomposition may be replaced by a suitably chosen simple ideal of the other. Thus by Theorem 3.3 and Corollary 3.1 we have the

KUROSCH-ORE DECOMPOSITION THEOREM. Let an element of a modular lattice have two reduced decompositions into irreducibles. Then the number of components in the two decompositions is the same and any component in one decomposition may be replaced by a suitably chosen component of the other.

4. Lattices with unique decompositions. This section will be devoted to the proof of the following theorem:

THEOREM 4.1. Let $\subseteq$ satisfy the ascending chain condition. Then each element of $\subseteq$ has a unique representation as a reduced cross-cut of irreducibles if and only if $\mathfrak{S}$ is a Birkhoff lattice and $\mathfrak{R}_{a}$ is a Boolean algebra for each a.

We begin with a series of lemmas, the first of which proves the necessity of the conditions of the theorem.

LEMMA 4.1. Let $\mathfrak{S}$ satisfy the ascending chain condition and let each element have a unique representation as a reduced cross-cut of irreducibles. Then $\subseteq$ is a Birkhoff lattice and $\mathfrak{R}_{a}$ is a Boolean algebra for each $a$.

For let $\mathfrak{b}>a, \mathfrak{c} \supset a$ and $\mathfrak{c} D \mathfrak{b}$. If $\mathfrak{b} \cup \mathfrak{c} \ngtr \mathfrak{c}$ we have $\mathfrak{b} \cup \mathfrak{c} \supset \mathfrak{b} \supset \mathfrak{c}$ where $\mathfrak{b} \cup \mathfrak{c} \neq \mathfrak{b} \neq \mathfrak{c}$. Since $\mathfrak{d} D \mathfrak{b}$, there exists a $d \in \mathfrak{d}$ such that $d \Phi \mathfrak{b}$. Since $\mathfrak{b} \neq \mathfrak{c}$, there exists a $c$ such that $c \in \mathfrak{c}, d \supset c$, and $c \nsupseteq d$. Furthermore since $c \nsupseteq d$ there exists an irreducible $q_{c}$ such that $q_{c} \supset c, q_{c} \supset \mathfrak{d}$ (Lemma 2.6). But then $\mathfrak{b} \supset \mathfrak{b} \cap q_{c} \supset a$ and if $\mathfrak{b}=\mathfrak{b} \cap q_{c}$ we have $q_{c} \supset \mathfrak{b} \cup \mathfrak{c} \supset \mathfrak{b}$ which contradicts $q_{c} D \mathfrak{b}$. Hence $a=\mathfrak{b} \cap q_{c}$. Similarly there exists an irreducible $q_{d}$ such that $q_{d} \supset d$ and $a=\mathfrak{b} \cap q_{d}$. By Theorem 2.2 we have $a=b_{c} \cap q_{c}$ and $a=b_{d} \cap q_{d}$ where $b_{c}, b_{d} \in \mathfrak{b}$. Let $b=b_{c} \cap b_{d}$. Then $b \in \mathfrak{b}$ and $a=b \cap q_{c}=b \cap q_{d}$. Let $b=q_{1} \cap \cdots \cap q_{k}$. Then $a$ has two reduced representations $a=q_{i_{1}} \cap \cdots \cap q_{i_{l}} \cap q_{c}=q_{j_{1}} \cap \cdots \cap q_{j_{m}} \cap q_{d}$. Now $q_{c} \neq q_{d}$ since otherwise $q_{c} \supset \mathfrak{d}$ and $q_{c} \neq q_{j_{r}}$ since otherwise $q_{c} \supset \mathfrak{b} \cup \mathfrak{c} \supset \mathfrak{b}$ contrary to $q_{c} D \mathfrak{b}$. Hence $a$ has two distinct reduced representations as a cross-cut of irreducibles which contradicts our hypothesis. Thus $\mathfrak{b} \cup \mathfrak{c}>\mathfrak{c}$ and hence each element of $\mathfrak{S}$ satisfies the Birkhoff condition in the lattice of ideals.

Now since each element has a unique decomposition into irreducibles, the number of components is obviously bounded and hence $\mathfrak{R}_{a}$ is archimedean by Theorem 3.1. Let $p_{1}, \cdots, p_{k}$ be a maximal independent set of point ideals of $\mathfrak{R}_{a}$. Then $\mathfrak{p}_{1}, \cdots, \mathfrak{p}_{k}$ generate a Boolean algebra with simple ideals $\boldsymbol{g}_{1}, \cdots, \mathbb{8}_{k}$. $\mathbb{z}_{1}, \cdots, \mathbb{z}_{k}$ are clearly simple ideals of $\mathfrak{R}_{a}$ and hence are characteristic ideals by Corollary 3.2. Thus $a$ has a decomposition $a=q_{1} \cap \cdots \cap q_{k}$ where $q_{i} \supset \Xi_{i}$ (Theorem 3.3). Now suppose there is a simple ideal 8 distinct from $\mathbb{8}_{1}, \cdots, \mathbb{8}_{k}$. Let $q \supset \varepsilon, q D \mathfrak{u}_{a}$. Then $q$ is a component of $a$ by Theorem 3.5 and hence $q=q_{i}$ for some $i$ since $a$ has but one reduced decomposition into irreducibles. But

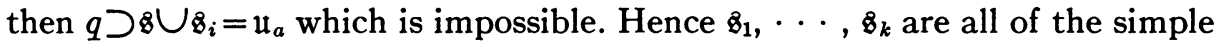


ideals of $\mathfrak{R}_{a}$ and since each ideal of $\mathfrak{R}_{a}$ can be expressed as a union cross-cut of simple ideals, $\mathfrak{R}_{a}$ is simply the Boolean algebra generated by $\mathfrak{p}_{1}, \cdots, \mathfrak{p}_{k}$.

\section{LEMma 4.2. If $\mathfrak{R}_{a}$ is a Boolean algebra, then it is archimedean.}

For if $\mathfrak{R}_{a}$ has an infinite number of point ideals, let $\mathfrak{p}_{1}, \mathfrak{p}_{2}, \mathfrak{p}_{3}, \cdots$ be a denumerable sequence of point ideals. Let $\mathfrak{p}_{i}^{\prime}=p_{1} \cup \mathfrak{p}_{2} \cup \ldots \cup \cup_{\mathfrak{p}_{i-1}} \cup \mathfrak{p}_{i+1} \cup \ldots$. Then since $\mathfrak{R}_{a}$ is a Boolean algebra we have $a=\mathfrak{p}_{1}^{\prime} \cap \mathfrak{p}_{2}^{\prime} \cap \cdots$. But since the cross-cut of an infinite number of ideals consists of all elements contained in finite cross-cuts $a=\mathfrak{p}_{1}^{\prime} \cap \mathfrak{p}_{2}^{\prime} \cap \cdots \cap \mathfrak{p}_{k}^{\prime}$ for some $k$. Then $a \supset \mathfrak{p}_{k+1}$ which contradicts $\mathfrak{p}_{k+1}>a$. Hence $\mathfrak{R}_{a}$ has only a finite number of point ideals and thus is archimedean.

LemMA 4.3. Let $\mathfrak{S}$ be a Birkhoff lattice in which each $\mathfrak{R}_{a}$ is archimedean. Then if every three ideals covering a principal ideal generate a Boolean algebra of order eight, $\mathbb{R}_{a}$ is a Boolean algebra for each $a$.

For let the hypotheses of the lemma be satisfied and let every three ideals covering a principal ideal generate a Boolean algebra. We show first that the ideals of any finite set of ideals covering a principal ideal are independent. Suppose that for any $a$ every $k-1$ ideals covering $a$ are independent. Let $\mathfrak{p}_{1}, \cdots, \mathfrak{p}_{k}$ be $k$ distinct ideals covering $a$. If $\mathfrak{p}_{1}, \cdots, \mathfrak{p}_{k}$ are not independent let $\mathfrak{p}_{1} \cup \mathfrak{p}_{2} \cup \ldots \cup \mathfrak{p}_{k-1} \supset \mathfrak{p}_{k}$ say. Now $\mathfrak{p}_{1} \cup \mathfrak{p}_{i} \supset \mathfrak{p}_{2}, \cdots, \mathfrak{p}_{i-1}, \mathfrak{p}_{i+1}, \cdots, \mathfrak{p}_{k}$ $(i=2, \cdots, k)$ since every three ideals covering $a$ generate a Boolean algebra. Hence elements $x_{i j} \in \mathfrak{p}_{1}$ exist such that $x_{i j} \cup \mathfrak{p}_{i} \supset_{\mathfrak{p}_{j}}(j=2, \cdots, i-1, i+1, \cdots, k$; $i=2, \cdots, k)$. Let $x=x_{23} \cap x_{24} \cap \cdots \cap x_{k k-1}$. Then $x \in p_{1}$ and $x \cup \mathfrak{p}_{i} D_{p_{2}}, \cdots$, $\mathfrak{p}_{i-1}, \mathfrak{p}_{i+1}, \cdots, \mathfrak{p}_{k}(i=2, \cdots, k)$. Clearly $x \nsupseteq \mathfrak{p}_{2}, \cdots, \mathfrak{p}_{k}$. Hence $\mathfrak{p}_{2}^{\prime}=x \cup \mathfrak{p}_{2}$ $>x, \cdots, \mathfrak{p}_{k}^{\prime}=x \cup \mathfrak{p}_{k}>x$ and $\mathfrak{p}_{2}^{\prime}, \cdots, \mathfrak{p}_{k}^{\prime}$ are distinct. Thus by the induction assumption $\mathfrak{p}_{2}^{\prime}, \cdots, \mathfrak{p}_{k}^{\prime}$ are independent. But $\mathfrak{p}_{2}^{\prime} \cup \ldots \cup \mathfrak{p}_{k-1}^{\prime} \supset x \cup \mathfrak{p}_{2} \cup \ldots$ $\cup_{\mathfrak{p}_{k-1}} \supset x \cup \mathfrak{p}_{1} \cup \ldots \cup \mathfrak{p}_{k-1} \supset x \cup \mathfrak{p}_{k}=\mathfrak{p}_{k}^{\prime}$ which is contrary to the independence. Hence the independence of any finite set of covering elements follows by induction.

Now let $a \in \Omega_{a}$ and let $\mathfrak{p}_{1}, \cdots, \mathfrak{p}_{k}$ be a maximal independent set of point ideals of $\mathfrak{R}_{a}$ divisible by $\mathfrak{a}$. Imbed $\mathfrak{p}_{1}, \cdots, \mathfrak{p}_{k}$ in a maximal independent set $\mathfrak{p}_{1}, \cdots, \mathfrak{p}_{k}, \cdots, \mathfrak{p}_{n}$. Set $\mathfrak{b}=\mathfrak{p}_{1} \cup \cdots \cup \mathfrak{p}_{k}$. Then $\mathfrak{a} \supset \mathfrak{b}$. If $\mathfrak{b} D \mathfrak{a}$, there exists an element $b_{1} \in \mathfrak{b}$ such that $b_{1} D \mathfrak{a}$. Now $\mathfrak{b} \cup \mathfrak{p}_{k+i} \triangleright \mathfrak{p}_{k+j}(j=k+1, \cdots, k+i-1$, $k+i+1, \cdots, n ; i=1, \cdots, n-k)$. Hence as above there exists an element $b_{2} \in \mathfrak{b}$ such that $b_{2} \cup \mathfrak{p}_{k+i} \perp \mathfrak{p}_{k+i}, i \neq j$. Also $\mathfrak{a}=\mathfrak{a} \cup \mathfrak{b} D \mathfrak{p}_{k+1}, \cdots, \mathfrak{p}_{n}$. Hence an element $b_{3} \in \mathfrak{b}$ exists such that $\mathfrak{a} \cup b_{3} \supseteq \mathfrak{p}_{k+1}, \cdots, \mathfrak{p}_{n}$. Set $b=b_{1} \cap b_{2} \cap b_{3}$. Then

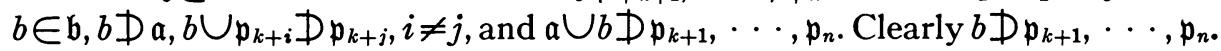
Hence $\mathfrak{p}_{k+1}^{\prime}=b \cup \mathfrak{p}_{k+1}>b, \cdots, \mathfrak{p}_{n}^{\prime}=b \cup \mathfrak{p}_{n}>b$ and $\mathfrak{p}_{k+1}^{\prime}, \cdots, \mathfrak{p}_{n}^{\prime}$ are distinct. Let $b \cup \mathfrak{a} \supset \mathfrak{p}>b$. Then $\mathfrak{p}$ is distinct from $\mathfrak{p}_{k+1}^{\prime}, \cdots, \mathfrak{p}_{n}^{\prime}$. For if $\mathfrak{p}=\mathfrak{p}_{k+i}^{\prime}$, then $b \cup \mathfrak{a} \supset \mathfrak{p}_{k+i}$ contrary to the definition of $b$. Thus by the result of the above paragraph $\mathfrak{p}, \mathfrak{p}_{k+1}^{\prime}, \cdots, \mathfrak{p}_{n}^{\prime}$ are independent. But $\mathfrak{p}_{k+1}^{\prime} \cup \ldots \cup \mathfrak{p}_{n}^{\prime}=b \cup \mathfrak{p}_{k+1}$ $\cup \ldots \cup \cup_{\mathfrak{p}_{n}}=b \cup \cup_{\mathfrak{p}_{1}} \cup \ldots \cup \cup_{\mathfrak{p}_{n}} \supset b \cup \mathfrak{a} \supset \mathfrak{p}$ which is impossible. Hence $\mathfrak{a}=\mathfrak{b}$ and 
$\mathfrak{R}_{a}$ is a point lettice. But then the point ideals of $\mathfrak{R}_{a}$ are independent and generate $\mathfrak{R}_{a}$. Thus $\mathfrak{R}_{a}$ is a Boolean algebra by Lemma 1.2.

LemMA 4.4. Let $\subseteq$ be a Birkhoff lattice satisfying the ascending chain condition in which every three ideals covering a principal ideal generate a Boolean algebra. Let $q$ be an irreducible of $\subseteq$ such that $q \supset a ; \mathfrak{b}, \mathfrak{c}>a$ and $\mathfrak{b} \neq \mathfrak{c}$. Then either $q \supset \mathfrak{b}$ or $q \supset \mathfrak{c}$.

Let us suppose that for some $a$ we have $q \supset a ; \mathfrak{b}, \mathfrak{c}>a, \mathfrak{b} \neq \mathfrak{c}, q D \mathfrak{b}$ and $q D \mathfrak{c}$. We shall show that a proper divisor $a^{\prime}$ of $a$ exists with the same properties and hence the lemma follows from the ascending chain condition. Now $q \neq a$ since otherwise $q=\mathfrak{b} \cap \mathfrak{c}$ contrary to the irreducibility of $q$. Hence $q \supset \mathfrak{p}>a$ by Theorem 2.1. Clearly $\mathfrak{p} \neq \mathfrak{b}, \mathfrak{c}$ since otherwise $q \supset \mathfrak{b}$ or $q \supset \mathfrak{c}$. Hence $\mathfrak{p}, \mathfrak{b}$ and $\mathfrak{c}$ generate a Boolean algebra. Since $\mathfrak{p} \cup \mathfrak{b} D \mathfrak{c}$ there exists an element $p \in \mathfrak{p}$ such that $p \cup \mathfrak{b} D \mathfrak{c}$. Since $q \supset \mathfrak{p}$, there exists an element $p^{\prime} \in \mathfrak{p}$ such that $q \supset p^{\prime}$. Let $a^{\prime}=p \cap p^{\prime}$. Then $a^{\prime} \in p$ and hence $a^{\prime} \neq a$. Clearly $q \supset a^{\prime}$. Let $\mathfrak{b}^{\prime}=a^{\prime} \cup \mathfrak{b}, \mathfrak{c}^{\prime}=a^{\prime} \cup \mathfrak{c}$. Then $\mathfrak{b}^{\prime}>a^{\prime}$ and $\mathfrak{c}^{\prime}>a^{\prime}$ by the Birkhoff condition. If $\mathfrak{b}^{\prime}=\mathfrak{c}^{\prime}$, then $p \cup \mathfrak{b} \supset a^{\prime} \cup \mathfrak{b}$ $\supset \mathfrak{c}$, which contradicts $p \cup \mathfrak{b} D \mathfrak{c}$. Hence $\mathfrak{b}^{\prime} \neq \mathfrak{c}^{\prime}$. Since $q \Phi \mathfrak{b}, q \Phi \mathfrak{c}$ we have $q \backslash \mathfrak{b}^{\prime}$, $q D c^{\prime}$. Thus $a^{\prime}$ is a proper divisor of $a$ with the desired properties.

LEMMA 4.5. Let $\subseteq$ be a Birkhoff lattice satisfying the ascending chain condition in which every three ideals covering a principal ideal generate a Boolean algebra. Then if a has a reduced representation $a=q_{1} \cap \cdots \cap q_{k}, \mathfrak{R}_{a}$ is archimedean of length $k$ and each $q_{i}$ divides a simple ideal of $\mathfrak{R}_{a}$.

For let $\mathfrak{a}_{i}$ be the union of the point ideals of $\mathfrak{R}_{a}$ which are divisible by $q_{i}$. Then $\mathfrak{a}_{i} \neq \mathfrak{u}_{a}$ since $\mathfrak{a}_{i}$ is a characteristic ideal of $\mathfrak{R}_{a}$. Now let $\mathfrak{p}, \mathfrak{p}^{\prime}$ be any two point ideals of $\mathfrak{R}_{a}$ which are not divisible by $\mathfrak{a}_{i}$. Then $\mathfrak{a}_{i} \cup \mathfrak{p}>\mathfrak{a}_{i}$ and $\mathfrak{a}_{i} \cup \mathfrak{p}^{\prime}>\mathfrak{a}_{i}$ by the Birkhoff condition. Now suppose that $a_{i} \cup \mathfrak{p} D \mathfrak{p}^{\prime}$. Then there exists an element $a_{1} \in \mathfrak{a}_{i}$ such that $a_{1} \cup \mathfrak{p} \supset \mathfrak{p}^{\prime}$. Since $q_{i} \supset \mathfrak{a}_{i}$, there exists an element $a_{2} \in \mathfrak{a}_{i}$ such that $q_{i} \supset a_{2}$. Let $a_{i}=a_{1} \cap a_{2}$. Then $q_{i} \supset a_{i}$ and $a_{i} \cup_{\mathfrak{p}} D \mathfrak{p}^{\prime}$. Clearly $a_{i} D \mathfrak{p}^{\prime}$. If $a_{i} \supset \mathfrak{p}$, then $q_{i} \supset \mathfrak{p}$ and $\mathfrak{a}_{i} \supset \mathfrak{p}$ contrary to assumption. Hence $a_{i} \cup_{\mathfrak{p}}>a_{i}$, $a_{i} \cup \mathfrak{p}^{\prime}>a_{i}$ and $a_{i} \cup \mathfrak{p} \neq a_{i} \cup \mathfrak{p}^{\prime}$. Since $q_{i} \supset a_{i}$ by Lemma 4.4 we have either $q_{i} \supset a_{i} \cup \mathfrak{p}$ or $q_{i} \supset a_{i} \cup \mathfrak{p}^{\prime}$. Hence $q_{i} \supset \mathfrak{p}$ or $q_{i} \supset \mathfrak{p}^{\prime}$. But then $\mathfrak{a}_{i} \supset \mathfrak{p}$ or $\mathfrak{a}_{i} \supset \mathfrak{p}^{\prime}$ contrary to assumption. Thus $\mathfrak{a}_{i} \cup \mathfrak{p} \supset \mathfrak{p}^{\prime}$ and $\mathfrak{a}_{i} \cup \mathfrak{p}=\mathfrak{a}_{i} \cup \mathfrak{p}^{\prime}$ for every pair of point ideals of $\mathfrak{R}_{a}$ not divisible by $\mathfrak{a}_{i}$. But then $\mathfrak{a}_{i} \cup \mathfrak{p}=\mathfrak{a}_{i} \cup \mathfrak{u}_{a}=\mathfrak{u}_{a}$ and $\mathfrak{u}_{a}>\mathfrak{a}_{i}$. Hence $\mathfrak{a}_{i}$ is simple and each $q_{i}$ divides a simple ideal of $\mathfrak{R}_{a}$.

Now let $\mathfrak{b}_{0}=\mathfrak{u}_{a}$ and let $\mathfrak{b}_{i}$ denote the union of the point ideals of $\mathfrak{R}_{a}$ which are divisible by $q_{1}, \cdots, q_{i}$. Then $\mathfrak{b}_{1}=\mathfrak{a}_{1}$ and $\mathfrak{b}_{0}>\mathfrak{a}_{1}$ by the result we have just obtained. Clearly $\mathfrak{b}_{l-1} \supset \mathfrak{b}_{l}$. If $\mathfrak{b}_{l-1}=\mathfrak{b}_{l}$, let $q_{1} \cap \cdots \cap q_{l-1} \cap q_{l+1} \cap \cdots \cap q_{k}$ $\supset \mathfrak{p}_{l}>a . p_{l}$ exists since the representation is reduced. Now $q_{1} \cap \cdots \cap q_{l-1} \supset p_{l}$ and hence $\mathfrak{b}_{l-1} \supset \mathfrak{p}_{l}$. But then $\mathfrak{b}_{l} \supset \mathfrak{p}_{l}$ and hence $q_{l} \supset \mathfrak{p}_{l}$. Thus $a=q_{1} \cap \cdots \cap q_{k} \supset \mathfrak{p}_{l}$ which is impossible. Hence $\mathfrak{b}_{l-1} \neq \mathfrak{b}_{l}$. Now let $\mathfrak{p}$ and $\mathfrak{p}^{\prime}$ be two point ideals divisible by $\mathfrak{b}_{l-1}$ but not by $\mathfrak{b}_{l}$. If $\mathfrak{b}_{l} \cup \mathfrak{p} \neq \mathfrak{b}_{l} \cup \mathfrak{p}^{\prime}$ there exists an element $b_{l} \in \mathfrak{b}_{l}$ such that $q_{l} \supset b_{l}, b_{l} \cup_{\mathfrak{p}}>b_{l}, b_{l} \cup_{\mathfrak{p}^{\prime}}>b_{l}$ and $b_{l} \cup_{\mathfrak{p}} \neq b_{l} \cup_{\mathfrak{p}^{\prime}}$. But then $q_{l} \supset b_{l} \cup_{\mathfrak{p}}$ 
or $q_{l} \supset b_{l} \cup \mathfrak{p}^{\prime}$ by Lemma 4.4. Hence either $\mathfrak{b}_{l} \supset \mathfrak{p}$ or $\mathfrak{b}_{l} \supset \mathfrak{p}^{\prime}$ which is contrary to assumption. Hence $\mathfrak{b}_{l} \cup_{\mathfrak{p}}=\mathfrak{b}_{l} \cup \cup_{\mathfrak{p}}$ for every two point ideals of $\mathfrak{b}_{l-1}$ which are not divisible by $\mathfrak{b}_{l}$. Thus $\mathfrak{b}_{l} \cup \mathfrak{p}=\mathfrak{b}_{l} \cup \mathfrak{b}_{l-1}=\mathfrak{b}_{l-1}$ and $\mathfrak{b}_{l-1}>\mathfrak{b}_{l}$ by the Birkhoff condition. Hence we have the chain $\mathfrak{u}_{a}>\mathfrak{b}_{1}>\mathfrak{b}_{2}>\cdots>\mathfrak{b}_{k}$. But $\mathfrak{b}_{k}=a$ and the lemma follows from Lemma 3.1.

LEMMA 4.6. Let $\subseteq$ be a Birkhoff lattice satisfying the ascending chain condition in which every three ideals covering a principal ideal generate a Boolean algebra. Then each $a \in \mathbb{S}$ has a unique reduced representation $a=q_{1} \cap \cdots \cap q_{k}$ where $q_{1}, \cdots, q_{k}$ are irreducibles. $\Omega_{a}$ is a Boolean algebra of order $2^{k}$ and each $q_{i}$ divides a simple ideal of $\mathfrak{R}_{a}$.

It follows from Lemmas 4.3 and 4.5 that $\Omega_{a}$ is a Boolean algebra of order $2^{k}$. $q_{i}$ divides a simple ideal $\xi_{i}$ of $\mathfrak{R}_{a}$ by Lemma 4.5 . Now let $a=q_{1}^{\prime} \cap \cdots \cap q_{l}^{\prime}$ be a reduced decomposition of $a$. By Lemma $4.5, l=k$ and $q_{i}^{\prime}$ divides a simple

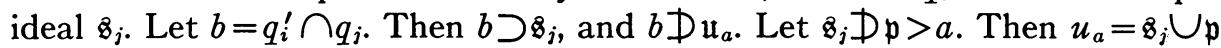
and $b \cup \mathfrak{p}=b \cup \mathbb{g}_{j} \cup \mathfrak{p}=b \cup \mathfrak{u}_{a}>b$ by the Birkhoff condition. If $q_{j} \neq b$, we have $q_{j} \supset \mathfrak{p}_{j}>b$ and $\mathfrak{p}_{j} \neq \mathfrak{p} \cup b$ since otherwise $q_{j} \supset \mathfrak{p} \cup \mathbb{B}_{j}=\mathfrak{u}_{a}$. Hence by Lemma 4.4, either $q_{i}^{\prime} \supset \mathfrak{p}_{j}$ or $q_{i}^{\prime} \supset \mathfrak{p}$. But if $q_{i}^{\prime} \supset \mathfrak{p}_{j}$, then $b=q_{i}^{\prime} \cap q_{j} \supset \mathfrak{p}_{j}>b$ which is impossible. Hence $q_{i}^{\prime} \supset \mathfrak{p}$ and $q_{i}^{\prime} \supset \mathfrak{u}_{a}$ which is impossible. Thus $q_{j}=b$ and similarly $q_{i}^{\prime}=b$. Hence $q_{i}^{\prime}$ is equal to $q_{j}$ and the two representations are identical. This completes the proof of the lemma.

Lemma 4.1 and Lemma 4.6 together give Theorem 4.1.

In view of Lemma 4.6, lattices with unique irreducible decompositions may be characterized in terms of the local properties of the lattice of ideals as follows:

THEOREM 4.2. Let $\subseteq$ satisfy the ascending chain condition. Then each element of $\subseteq$ has a unique reduced decomposition into irreducibles if and only if $\mathfrak{S}$ is a Birkhoff lattice in which every three ideals covering an element of $\subseteq$ are independent.

As a corollary to Lemma 4.6 we have

COROLlaRY 4.1. Let $\mathfrak{S}$ satisfy the ascending chain condition and let every element of $\mathfrak{S}$ have a unique reduced decomposition into irreducibles. Then the number of irreducible components of $a$ is equal to the number of ideals covering $a$.

COROLlARY 4.2. Let $\mathfrak{S}$ be a Birkhoff lattice satisfying the ascending chain condition. Then if $\mathbb{S}$ contains a modular, non-distributive sublattice, the lattice of ideals of 5 contains a complete $\left({ }^{10}\right)$ modular, non-distributive sublattice of order five.

For if $\subseteq$ contains a modular, non-distributive sublattice of order five, at

(10) A sublattice $\mathfrak{R}^{\prime}$ of $\mathfrak{R}$ is said to be complete if $\mathfrak{a}>\mathfrak{b}$ in $\mathfrak{R}^{\prime}$ implies $\mathfrak{a}>\mathfrak{b}$ in $\mathfrak{Q}$. 
least one element of $\subseteq$ does not have a unique decomposition into irreducibles. But then there are three ideals covering a principal ideal which are dependent. These three ideals generate a complete, modular, non-distributive sublattice of $\mathfrak{R}$ of order five.

5. Unicity of the number of components. In the previous section lattices with unique irreducible decompositions were completely characterized as Birkhoff lattices with certain special properties. Simple examples show that a similar characterization of lattices in which the number of components is unique will require lattices that are considerably more general than Birkhoff lattices. Hence we shall restrict ourselves to the characterization of Birkhoff lattices having the number of components unique. We prove the following theorem:

THEOREM 5.1. Let $\subseteq$ be a Birkhoff lattice satisfying the ascending chain condition. Then the number of components in the reduced decompositions of each element into irreducibles is unique if and only if $\mathfrak{R}_{a}$ is modular for each $a$.

As in $\$ 4$, the proof rests on a series of lemmas.

LEMma 5.1. Let $\subseteq$ be a Birkhoff lattice satisfying the ascending condition. Then the number of components in the irreducible decompositions of a is unique if and only if $\mathfrak{R}_{a}$ is archimedean, modular, and every characteristic ideal of $\mathfrak{R}_{a}$ is simple.

Since the ascending chain condition holds each element of $\subseteq$ has a decomposition into irreducibles. Now if the number of components in the irreducible decompositions of $a$ is unique it is certainly bounded and hence $\mathfrak{R}_{a}$ is archimedean by Theorem 3.1. Now let $c$ be a characteristic ideal of $\mathfrak{R}_{a}$ and let $\mathfrak{p}_{1}, \cdots, \mathfrak{p}_{k}$ be a maximal independent set of point ideals divisible by $c$. Imbed $\mathfrak{p}_{1}, \cdots, \mathfrak{p}_{k}$ in a maximal independent set $\mathfrak{p}_{1}, \cdots, \mathfrak{p}_{k}, \cdots, \mathfrak{p}_{n}$. By Theorem 3.5 and Theorem 3.3, $a$ has an irreducible decomposition having $k+1$ components. But by Corollary $3.2 a$ has a decomposition having $n$ components. Hence if the number of components is unique we have $n=k+1$. But then $\mathfrak{p}_{1} \cup \ldots \cup \mathfrak{p}_{k}$ is a simple ideal of $\mathfrak{R}_{a}$ and $\mathfrak{u}_{a} \supset \mathfrak{c} \supset \mathfrak{p}_{1} \cup \ldots \cup \mathfrak{p}_{k}, \mathfrak{u}_{a} \neq \mathfrak{c}$. Hence $\mathfrak{c}=\mathfrak{p}_{1} \cup \ldots \cup \mathfrak{p}_{k}$ and $\mathrm{c}$ is a simple ideal of $\mathfrak{R}_{a}$.

Now let $\mathfrak{z}$ be an arbitrary simple ideal of $\mathfrak{R}_{a}$ and let $\mathfrak{a}$ be any ideal of $\mathfrak{R}_{a}$ such that $\mathbb{Z} \mathfrak{D}$. By Theorem 3.2, $\mathfrak{a}$ has a reduced representation $\mathfrak{a}=\mathfrak{z}_{1} \cap \cdots \cap \mathfrak{z}_{l}$ where $\mathfrak{z}_{1}, \cdots, \mathfrak{z}_{l}$ are simple ideals of $\mathfrak{R}_{a}$. If $\mathfrak{a} \cap \mathbb{z} \neq a$, by Theorem 3.2 there exists a simple ideal $\mathbb{z}_{l+2}$ such that $\boldsymbol{z}_{l+2} D a \cap z$. Similarly if $a \cap \mathfrak{z} \cap z_{l+2} \neq a$, there exists a simple ideal $8_{l+3}$ such that $z_{l+3} D a \cap z \cap z_{l+2}$. Thus we eventually have $\mathfrak{a} \cap \mathrm{z}_{\mathrm{z}} \mathrm{z}_{l+2} \cap \cdots \cap \mathrm{z}_{m}=a$. Then $a=\mathrm{z}_{1} \cap \cdots \cap \mathrm{z}_{l} \cap \mathrm{z} \cap \mathrm{z}_{l+2} \cap \cdots \cap \mathrm{z}_{m}$ and since each simple ideal is characteristic this decomposition gives a decomposition into irreducibles with the same number of terms. Hence if the number of components in the irreducible decompositions of $a$ is unique we have $m \geqq n$ where $n$ is the length of $\mathfrak{z}_{a}$. But $\mathfrak{u}_{a} \supset \mathfrak{z}_{1} \supset \mathfrak{z}_{1} \cap \mathfrak{z}_{2} \supset \cdots \supset \mathfrak{z}_{1} \cap \cdots \cap \mathfrak{z}_{l} \supset \mathfrak{z}_{1}$ 
$\cap \cdots \cap \mathrm{z}_{l} \cap \mathrm{z} \supset \mathrm{z}_{1} \cap \cdots \cap \mathrm{z}_{l} \cap \mathrm{z}_{\mathrm{z}} \cap \mathrm{z}_{l+2} \supset \cdots \supset \mathrm{z}_{1} \cap \cdots \cap \mathrm{z}_{m}=a$ and the ideals of this chain are distinct. Hence $m \leqq n$ by Lemma 3.1. Thus $m=n$ and each ideal of the chain covers the ideal which immediately follows. Hence $\mathfrak{a}>\mathfrak{a} \cap \mathfrak{z}$. Now let $\mathfrak{a}$ and $\mathfrak{b}$ be any two ideals of $\mathfrak{R}_{a}$ such that $\mathfrak{a} \cup \mathfrak{b}>\mathfrak{b}$. By Theorem 3.2 and ideal $\mathfrak{z}$ exists such that $\mathfrak{z} \supset \mathfrak{b}, \mathfrak{B} D \mathfrak{a} \cup \mathfrak{b}$. But then $\mathfrak{b}=(\mathfrak{a} \cup \mathfrak{b}) \cap \mathbb{z}$. Hence $\mathfrak{a}>\mathfrak{a} \cap \mathfrak{z}=\mathfrak{a} \cap(\mathfrak{a} \cup \mathfrak{b}) \cap \mathfrak{z}=\mathfrak{a} \cap \mathfrak{b}$. Thus $\mathfrak{a} \cup \mathfrak{b}>\mathfrak{b}$ implies $\mathfrak{a}>\mathfrak{a} \cap \mathfrak{b}$ and B2 holds in $\mathfrak{R}_{a}$. But then $\mathfrak{R}_{a}$ is modular by Lemma 3.2.

On the other hand let $\mathfrak{R}_{a}$ be archimedean, modular, and every characteristic ideal be simple. Let $a=q_{1} \cap \cdots \cap q_{k}$ be a reduced decomposition into irreducibles. By Theorem 3.3, $a$ has a reduced representation $a=\mathfrak{c}_{1} \cap \cdots \cap \mathfrak{c}_{k}$ where $\mathfrak{c}_{i}$ is a characteristic ideal of $\mathfrak{R}_{a}$. But then $\mathfrak{c}_{i}$ is a simple ideal of $\mathfrak{R}_{a}$ by assumption. Thus $\mathfrak{u}_{a}>\mathfrak{c}_{1}>\mathfrak{c}_{1} \cap \mathfrak{c}_{2}>\cdots>\mathfrak{c}_{1} \cap \cdots \cap \mathfrak{c}_{k}=a$ since B2 holds in $\mathfrak{R}_{a}$ by Lemma 1.5. Hence $k$ is simply the length of $\mathfrak{R}_{a}$ and every reduced decomposition of $a$ into irreducibles has the same number of components. This completes the proof of the lemma.

Lemma 5.2. Let $\subseteq$ be a Birkhoff lattice satisfying the ascending chain condition. Then if $\mathfrak{R}_{a}$ is modular for each $a$, every characteristic ideal of $\mathfrak{R}_{a}$ is simple.

Let every characteristic ideal of $\mathfrak{R}_{b}$ be simple for every proper divisor $b$ of $a$. We shall show that every characteristic ideal of $\mathfrak{R}_{a}$ is simple and the lemma follows by the ascending chain condition.

If $\mathfrak{c}$ is a characteristic ideal of $\mathfrak{R}_{a}$ which is not simple, let $q$ be an associated irreducible. If $x$ is any element of $\subseteq$ divisible by $q$, let $\mathfrak{q}_{x}$ denote the union of the point ideals of $\mathfrak{R}_{x}$ divisible by $q$. Then since $\mathrm{c}$ is a characteristic ideal associated with $q$ we have $\mathfrak{c} \supset \mathfrak{q}_{a}$ and hence $\mathfrak{q}_{a}$ is not a simple ideal of $\mathfrak{R}_{a}$. Now suppose that for every two point ideals $\mathfrak{p}$ and $\mathfrak{p}^{\prime}$ such that $\mathfrak{q}_{a} D \mathfrak{p}, \mathfrak{p}^{\prime}$ we have $\mathfrak{q}_{a} \cup \mathfrak{p}=\mathfrak{q}_{a} \cup \mathfrak{p}^{\prime}$. Then $\mathfrak{u}_{a}=\mathfrak{q}_{a} \cup \mathfrak{u}_{a}=\mathfrak{q}_{a} \cup \mathfrak{p}>\mathfrak{q}_{a}$ and $\mathfrak{q}_{a}$ is simple contrary to assumption. Hence there are two point ideals $\mathfrak{p}$ and $\mathfrak{p}^{\prime}$ such that $\mathfrak{q}_{a} D \mathfrak{p}, \mathfrak{q}_{a} D \mathfrak{p}^{\prime}$, and $\mathfrak{q}_{a} \cup \mathfrak{p} \neq \mathfrak{q}_{a} \cup \mathfrak{p}^{\prime}$. Now $q \cap\left(\mathfrak{q}_{a} \cup \mathfrak{p} \cup \mathfrak{p}^{\prime}\right)=\left(q \cap \mathfrak{u}_{a}\right) \cap\left(\mathfrak{q}_{a} \cup \mathfrak{p} \cup \mathfrak{p}^{\prime}\right)=\mathfrak{q}_{a} \cup\left(q \cap \mathfrak{u}_{a}\right.$ $\left.\cap\left(\mathfrak{p} \cup \mathfrak{p}^{\prime}\right)\right)=\mathfrak{q}_{a} \cup\left(q \cap\left(\mathfrak{p} \cup \mathfrak{p}^{\prime}\right)\right)$ since $\mathbb{R}_{a}$ is modular. If $q \cap\left(\mathfrak{p} \cup \mathfrak{p}^{\prime}\right) \neq a$, we have $q \cap\left(\mathfrak{p} \cup \mathfrak{p}^{\prime}\right) \supset \mathfrak{p}_{1}>a$. If $\mathfrak{p}^{\prime}=\mathfrak{p}_{1}$, we have $q \supset \mathfrak{p}^{\prime}$ and hence $\mathfrak{q}_{a} \supset \mathfrak{p}^{\prime}$ contrary to hypothesis. Thus $\mathfrak{p}_{1} \neq \mathfrak{p}$ and $\mathfrak{p}_{1} \neq \mathfrak{p}^{\prime}$. Now $\mathfrak{p} \cup \mathfrak{p}^{\prime} \supset \mathfrak{p} \cup \mathfrak{p}_{1} \supset \mathfrak{p}$ and $\mathfrak{p} \cup \mathfrak{p}_{1} \neq \mathfrak{p}$. Hence $\mathfrak{p} \cup \mathfrak{p}^{\prime}=\mathfrak{p} \cup \mathfrak{p}_{1}$ by the Birkhoff condition. Since $q \supset \mathfrak{p}_{1}$ we have $q_{a} \supset \mathfrak{p}_{1}$ and hence $\mathfrak{q}_{a} \cup \mathfrak{p} \supset \mathfrak{p}_{1} \cup \mathfrak{p} \supset \mathfrak{p}^{\prime}$. But then $\mathfrak{q}_{a} \cup \mathfrak{p}=\mathfrak{q}_{a} \cup \mathfrak{p}^{\prime}$ which contradicts the definition of $\mathfrak{p}$ and $\mathfrak{p}^{\prime}$. Thus $q \cap\left(\mathfrak{p} \cup \mathfrak{p}^{\prime}\right)=a$ and $q \cap\left(\mathfrak{q}_{a} \cup \mathfrak{p} \cup \mathfrak{p}^{\prime}\right)=\mathfrak{q}_{a}$.

Now suppose that $q_{a}$ is not principal. Let $X$ be the set of all elements $x$ such that $q \supset x \supset \mathfrak{q}_{a}, q \neq x$. If $x \in X$, let $\mathfrak{p}_{x}=q \cap\left(x \cup \mathfrak{p} \cup \mathfrak{p}^{\prime}\right)$. Clearly $X$ generates $\mathfrak{q}_{a}$. We shall show

(1) There exists an $x_{0} \in X$ such that $x \cup \mathfrak{p} \cup \mathfrak{p}^{\prime}>\mathfrak{p}_{x}>x$ for all $x \in X, x_{0} \supset x$.

(2) The set of ideals $\mathfrak{p}_{x}, x \in X, x_{0} \supset x$, generates $\mathfrak{q}_{a}$.

(1) Since $\mathfrak{q}_{a} \cup \mathfrak{p} D \mathfrak{p}^{\prime}$ and $X$ generates $\mathfrak{q}_{a}$, there exists an element $x_{0} \in X$ such that $x_{0} \cup \mathfrak{p} D \mathfrak{p}^{\prime}$. Let $x_{0} \supset x, x \in X$ and suppose that $x=\mathfrak{p}_{x}$. Since $x$ is a proper divisor of $a$ we have $\mathfrak{u}_{x}>\mathfrak{q}_{x}$. By the Birkhoff condition $x \cup_{\mathfrak{p}}>x$, 
$x \cup_{\mathfrak{p}^{\prime}}>x$ and hence $x \cup_{\mathfrak{p}}, x \cup_{\mathfrak{p}^{\prime}}$ belong to $\mathfrak{R}_{x}$. Now $\mathfrak{q}_{x} D x \cup_{\mathfrak{p}} \cup_{\mathfrak{p}^{\prime}}$ since otherwise $q \supset \mathfrak{p}$. Hence by the modularity of $\mathfrak{R}_{x}$ we have $x \cup_{\mathfrak{p}} \cup_{\mathfrak{p}^{\prime}}>\mathfrak{q}_{x} \cap\left(x \cup \mathfrak{p} \cup \mathfrak{p}^{\prime}\right)$. Then $x \cup_{\mathfrak{p}} \cup_{\mathfrak{p}^{\prime}} \supset q \cap\left(x \cup_{\mathfrak{p}} \cup_{\mathfrak{p}^{\prime}}\right) \supset \mathfrak{q}_{x} \cap\left(x \cup_{\mathfrak{p}} \cup_{\mathfrak{p}^{\prime}}\right)$ and $q \cap\left(x \cup_{\mathfrak{p}} \cup_{\mathfrak{p}^{\prime}}\right) \neq x \cup_{\mathfrak{p}} \cup_{\mathfrak{p}^{\prime}}$. Thus $x=\mathfrak{p}_{x}=q \cap\left(x \cup \mathfrak{p} \cup \mathfrak{p}^{\prime}\right)=\mathfrak{q}_{x} \cap\left(x \cup \mathfrak{p} \cup \mathfrak{p}^{\prime}\right)$ and hence $x \cup \mathfrak{p} \cup \mathfrak{p}^{\prime}>x$. But then $x \cup_{\mathfrak{p}} \cup_{\mathfrak{p}^{\prime}} \supset x \cup \mathfrak{p} \supset x$ and if $x=x \cup_{\mathfrak{p}}$ we have $q \supset \mathfrak{p}$ which is impossible. Thus $x \cup_{\mathfrak{p}}=x \cup_{\mathfrak{p}} \cup_{\mathfrak{p}^{\prime}} \supset \mathfrak{p}^{\prime}$ and $x_{0} \cup_{\mathfrak{p}} \supset x \cup_{\mathfrak{p}} \supset \mathfrak{p}^{\prime}$ contrary to the definition of $x_{0}$. Hence $x \neq \mathfrak{p}_{x}$. Let $\mathfrak{p}_{x} \supset \mathfrak{p}_{x}^{\prime}>x$. Clearly $x \cup_{\mathfrak{p}} \cup_{\mathfrak{p}^{\prime}} \supset x \cup \mathfrak{p} \cup \mathfrak{p}_{x}^{\prime} \supset x \cup_{\mathfrak{p}}$ and $x \cup_{\mathfrak{p}} \cup_{\mathfrak{p}^{\prime}}$ $>x \cup \mathfrak{p}$ by the Birkhoff condition. If $x \cup_{\mathfrak{p}} \cup_{p_{x}^{\prime}}=x \cup_{\mathfrak{p}}$ we have $x \cup_{\mathfrak{p}} \supset \mathfrak{p}_{x}^{\prime}$ and $x=q \cap(x \cup \mathfrak{p}): \mathfrak{p}_{x}^{\prime}$ which contradicts $\mathfrak{p}_{x}^{\prime}>x$. Hence $x \cup_{\mathfrak{p}} \cup_{\mathfrak{p}^{\prime}}=x \cup_{\mathfrak{p}} \cup_{\mathfrak{p}_{x}^{\prime}}$. But then $x \cup \mathfrak{p} \cup \mathfrak{p}_{x}^{\prime} \supset \mathfrak{p}_{x} \supset \mathfrak{p}_{x}^{\prime}$ and $x \cup \mathfrak{p} \cup \mathfrak{p}_{x}^{\prime}>\mathfrak{p}_{x}^{\prime}$. If $x \cup \mathfrak{p} \cup \mathfrak{p}_{x}^{\prime}=\mathfrak{p}_{x}$, then $q \supset x \cup \mathfrak{p} \cup \mathfrak{p}^{\prime}$ which is impossible. Hence $\mathfrak{p}_{x}=\mathfrak{p}_{x}^{\prime}$ and $x \cup \mathfrak{p} \cup \mathfrak{p}^{\prime}>\mathfrak{p}_{x}>x$.

(2) Clearly $\mathfrak{p}_{x} \supset \mathfrak{q}_{a}$ for every $x$ since $\mathfrak{p}_{x} \supset x \supset \mathfrak{q}_{a}$. Now let $a_{1} \in \mathfrak{q}_{a}$. Then $a_{1} \supset \mathfrak{q}_{a}=q \cap\left(\mathfrak{q}_{a} \cup \mathfrak{p} \cup \mathfrak{p}^{\prime}\right)$ and hence $a_{1} \supset q \cap\left(a_{2} \cup \mathfrak{p} \cup \mathfrak{p}^{\prime}\right)$ where $a_{2} \in \mathfrak{q}_{a}$ by Theorem 2.2. Let $x=x_{0} \cap a_{2}$. Then $x \in X$ and $a_{1} \supset q \cap\left(x \cup \mathfrak{p} \cup \mathfrak{p}^{\prime}\right)=\mathfrak{p}_{x}$. Hence each element of $\mathfrak{q}_{a}$ divides some $\mathfrak{p}_{x}$ and thus the ideals $\mathfrak{p}_{x}$ generate $\mathfrak{q}_{a}$.

Now let $y$ be an arbitrary element of $\mathfrak{q}_{a} \cup_{\mathfrak{p}}$. Then $y \supset \mathfrak{q}_{a}$ and hence $y \supset \mathfrak{p}_{x}$ where $x_{0} \supset x$ by (2). But then by (1) $x \cup \mathfrak{p} \cup \mathfrak{p}^{\prime}>\mathfrak{p}_{x}>x$ and $\mathfrak{p}_{x} D \mathfrak{p}$ since otherwise $q \supset \mathfrak{p}$. Now $x \cup \mathfrak{p} \cup \mathfrak{p}^{\prime} \supset \mathfrak{p}_{x} \cup \mathfrak{p} \supset \mathfrak{p}_{x}$ and $\mathfrak{p}_{x} \cup \mathfrak{p} \neq \mathfrak{p}_{x}$. Hence $x \cup \mathfrak{p} \cup \mathfrak{p}^{\prime}=\mathfrak{p}_{x} \cup \mathfrak{p}$ which gives $\mathfrak{p}_{x} \cup \mathfrak{p} \supset \mathfrak{p}^{\prime}$. Thus $y \cup \mathfrak{p} \supset \mathfrak{p}^{\prime}$ for every $y$ and hence $\mathfrak{q}_{a} \cup_{\mathfrak{p}} \supset \mathfrak{p}^{\prime}$. The assumption that $\mathfrak{q}_{a}$ is not principal has thus led to a contradiction and we conclude that $\mathfrak{q}_{a}$ is principal, say $\mathfrak{q}_{a}=\left(a_{1}\right)$. Since $a_{1}$ is a proper divisor of $a$, by hypothesis we have $\mathfrak{u}_{a_{1}}>\mathfrak{q}_{a_{1}}$. Hence $\mathfrak{q}_{a_{1}} \cup\left(a_{1} \cup \mathfrak{p} \cup \mathfrak{p}^{\prime}\right)>\mathfrak{q}_{a_{1}}$. But $\mathfrak{q}_{a_{1}} \cap\left(a_{1} \cup \mathfrak{p} \cup \mathfrak{p}^{\prime}\right)$ $=q \cap\left(a_{1} \cup \mathfrak{p} \cup \mathfrak{p}^{\prime}\right)=a_{1}$ and $a_{1} \cup \mathfrak{p} \cup \mathfrak{p}^{\prime} \ngtr a_{1}$. Hence $\mathfrak{R}_{a_{1}}$ is non-modular contrary to assumption. Thus $\mathfrak{q}_{a}$ is simple and hence $\mathfrak{c}$ is a simple ideal of $\mathfrak{R}_{a}$.

Lemma 5.3. Let $\mathfrak{S}$ be a Birkhoff lattice satisfying the ascending chain condition. Then if $\mathfrak{R}_{a}$ is modular for every $a, \mathfrak{R}_{a}$ is archimedean.

For let $a=q_{1} \cap \cdots \cap q_{n}$ be a reduced decomposition of a into irreducibles. Then $a$ has the reduced representation $a=\mathfrak{c}_{1} \cap \cdots \cap c_{k}$ where $\mathfrak{c}_{i}$ is a characteristic ideal associated with $q_{i}$. By Lemma $5.2, c_{i}$ is a simple ideal of $\mathfrak{R}_{a}$. Hence since $\mathfrak{R}_{a}$ is modular we have $\mathfrak{u}_{a}>\mathfrak{c}_{1}>\mathfrak{c}_{1} \cap \mathfrak{c}_{2}>\cdots>\mathfrak{c}_{1} \cap \cdots \cap \mathfrak{c}_{k}=a$. Thus $\mathfrak{R}_{a}$ is archimedean of length $k$.

Lemmas 5.1-5.3 together give Theorem 5.1.

COROLlary 5.1. Let $\mathfrak{S}$ be a Birkhoff lattice satisfying the ascending chain condition. Let the number of components in the reduced decompositions of an element a be unique. Then in any two reduced decompositions of a, each component of one decomposition may be replaced by a suitably chosen component of the other.

For by Lemma 5.1, the two decompositions give two reduced representations of $a$ as a cross-cut of simple ideals of $\mathfrak{R}_{a}$. However, since $\mathfrak{R}_{a}$ is modular, B2 is satisfied and the replacement property follows from the dual of Lemma 1.3 . 
COROLla RY 5.2. Let $\mathfrak{S}$ be a Birkhoff lattice satisfying the ascending chain condition. Then if the number of components in the decompositions of an element a is unique, that number is simply the length of $\mathfrak{R}_{a}$.

COROLLARY 5.3. Let $\mathfrak{\subseteq}$ be a complemented Birkhoff lattice in which every element can be expressed as a cross-cut of a finite number of irreducibles. Then the number of components in the reduced decompositions of the null element $z$ is unique if and only if $\subseteq$ is a complemented modular lattice of finite dimensions.

For since $\mathfrak{S}$ is complemented, $\mathbb{R}_{z}$ is simply $\mathfrak{R}$, the lattice of ideals.

6. The Mac Lane exchange axiom. In order to free condition B1 of the covering properties, Mac Lane (Mac Lane [1]) formulated the following axiom.

$\mathrm{E}_{5}$. If $a \supset b \supset a \cap c$ and $c \neq a \cap c$, then there exists an element $c_{1} \neq \dot{a} \cap c$ such that $c \supset c_{1} \supset a \cap c$ and $b=a \cap\left(b \cup c_{1}\right)$.

Mac Lane showed that $E_{5}$ is equivalent to a transposition property of chains and in case covering elements exist, that is, if $b \supset a, b \neq a$, implies $b^{\prime}$ exists such that $b \supset b^{\prime}>a$, it reduces to $\mathrm{B} 1$. Thus both $\mathrm{E}_{5}$ and the requirement that each element satisfy the Birkhoff condition in the lattice of ideals are generalizations of $\mathrm{B} 1$. We shall be particularly interested in the conditions under which they are equivalent.

TheOREM 6.1. Every Birkhoff lattice satisfies $\mathrm{E}_{5}$.

Proof. Let $a \supset b \supset a \cap c$ and $c \neq a \cap c$. Then by Theorem 2.1 and ideal $\mathfrak{p}$ exists such that $c \supset \mathfrak{p}>a \cap b$. Now $b D_{p}$ since otherwise $a \cap c \supset \mathfrak{p}$ which is impossible. Hence $b \cup_{\mathfrak{p}}>b$ by the Birkhoff condition. But then $b \cup_{\mathfrak{p}} \supset a \cap(b \cup \mathfrak{p})$ $\supset b$ and if $b \cup \mathfrak{p}=a \cap(b \cup \mathfrak{p})$ we have $a \supset \mathfrak{p}$ which is impossible. Hence $b=a \cap(b \cup \mathfrak{p})$. Thus by Theorem 2.2 an element $p \in \mathfrak{p}$ exists such that $b=a \cap(b \cup p)$. Let $c_{1}=c \cap p$. Then $c \supset c_{1} \supset p>a \cap c$ and hence $c \supset c_{1} \supset a \cap c$, $c_{1} \neq a \cap c$. Also $b=a \cap(b \cup p) \supset a \cap\left(b \cup c_{1}\right) \supset b$. Thus $b=a \cap\left(b \cup c_{1}\right)$ and $c_{1}$ satisfies the requirements of $E_{5}$.

THEOREM 6.2. Let $\mathfrak{S}$ satisfy $\mathrm{E}_{5}$ and have the property that each element is covered by only a finite number of covering ideals. Then $\subseteq$ is a Birkhoff lattice.

Proof. Let $\mathfrak{a} \supset a, \mathfrak{p}>a$ and $\mathfrak{a} \supset \mathfrak{p}$. Let $\mathfrak{p}, \mathfrak{p}_{1}, \cdots, \mathfrak{p}_{n}$ be the finite number of ideals covering $a$. Now if $\mathfrak{p} \cup \mathfrak{a} \ngtr \mathfrak{a}$, we have $\mathfrak{p} \cup \mathfrak{a} \supset \mathfrak{c} \supset \mathfrak{a}, \mathfrak{p} \cup \mathfrak{a} \neq \mathfrak{c} \neq \mathfrak{a}$. Since $\mathfrak{p} \supset \mathfrak{p} \cap \mathfrak{c} \supset a$ and $\mathfrak{c} D \mathfrak{p}$ we have $\mathfrak{p} \cap \mathfrak{c}=a$ and hence by Theorem 2.2 elements $p^{\prime} \in \mathfrak{p}$ and $c \in \mathfrak{c}$ exist such that $p^{\prime} \cap c=a$. Since $c \neq a$, there exists an element $b^{\prime} \in \mathfrak{a}$ such that $b^{\prime} D \mathfrak{c}$. Let $b=c \cap b^{\prime}$. Then $b D c$ and $c \supset b \supset c \cap p^{\prime}$. Now since $p D p_{i}(i=1, \cdots, n)$ elements $p_{i}^{\prime}$ exist such that $p_{i}^{\prime} \in \mathfrak{p}$ and $p_{i}^{\prime} D p_{i}$ $(i=1, \cdots, n)$. Set $p=p^{\prime} \cap p_{1}^{\prime} \cap \cdots \cap p_{n}^{\prime}$. Then $p \in p, p^{\prime} \supset p$ and $p D p_{i}$ $(i=1, \cdots, n)$. Clearly $a=c \cap p^{\prime} \supset c \cap p \supset a$ implies $c \cap p=a$. Hence $c \supset b \supset c \cap p$ and $p \neq c \cap p$. Thus by $E_{5}$ an element $p_{1}$ exists such that $p_{1} \neq a, p \supset p_{1} \supset a$ and 
$b=c \bigcap\left(b \cup p_{1}\right)$. Now $p_{1} \supset \mathfrak{p}^{\prime}>a$ and $\mathfrak{p}^{\prime} \neq \mathfrak{p}_{i}(i=1, \cdots, n)$ since otherwise $p \supset p_{1} \supset \mathfrak{p}_{i}$ contrary to the definition of $p$. Hence $\mathfrak{p}^{\prime}=\mathfrak{p}$. But then $b=c \cap\left(b \cup p_{1}\right)$ $\supset \mathfrak{c} \cap(\mathfrak{a} \cup \mathfrak{p}) \supset \mathfrak{c}$ which contradicts $b \supset \mathfrak{c}$. Hence $\mathfrak{p} \cup \mathfrak{a}>\mathfrak{a}$ and $\mathfrak{S}$ is a Birkhoff lattice.

Now by Lemma 4.6, if $\mathfrak{S}$ is a Birkhoff lattice in which the ascending chain condition holds and every three ideals covering a principal ideal generate a Boolean algebra, then each $a$ is covered by only a finite number of ideals. However, Theorem 6.2 does not enable us to replace the Birkhoff condition in the lemma by $E_{5}$ since the proof of the finiteness required the Birkhoff condition. To carry out this replacement we first replace the condition that every three ideals covering a principal ideal generate a Boolean algebra by an equivalent condition.

THEÓREM 6.3. Let $\subseteq$ be a Birkhoff lattice satisfying the ascending chain condition. Then every three ideals covering a principal ideal generate a Boolean algebra if and only if $a \cup b \supset \mathfrak{q}>a \cap b$ implies $a \supset \mathfrak{q}$ or $b \supset \mathfrak{q}$.

Proof. Let every three ideals covering a principal ideal generate a Boolean algebra and suppose that $a \cup b \supset \mathfrak{q}>a \cap b$ but $a \nsupseteq \mathfrak{q}, b D \mathfrak{q}$. Then $a, b \neq a \cap b$. For if $a=a \cap b$, then $b=a \cup b \supset \mathfrak{q}$ contrary to assumption. Now with $b$ fixed let $a$ be maximal such that $a \cup b \supset \mathfrak{q}>a \cap b$ and $a \perp \mathfrak{q}, b D \mathfrak{q}$ for some $\mathfrak{q}$. Let $b \supset \mathfrak{p}>a \cap b$. Suppose $\mathfrak{a}=\mathfrak{p} \cup a \supset \mathfrak{q}$. Then $\mathfrak{a} \cup b=a \cup \mathfrak{p} \cup b=a \cup b \supset \mathfrak{q}$ and $\mathfrak{a} \cap b$ $\supset a \cap b$. Now $\mathfrak{a} \neq a$ since otherwise $a \supset \mathfrak{p}$ and $a \cap b \supset \mathfrak{p}>a \cap b$ which is impossible. Let $a_{1}^{\prime}$ be an element of $\mathfrak{a}$ such that $a_{1}^{\prime} D \mathfrak{q}$. Now $a \cup b \supset b \supset \mathfrak{p}$ and hence $a \cup b \supset \mathfrak{p} \cup a=\mathfrak{a}$. Thus $a_{1}=(a \cup b) \cap a_{1}^{\prime} \supset \mathfrak{a}$ and $a \cup b \supset a_{1}$. But $a \cup b \supset a_{1} \cup b \supset a$ $\cup b$. Hence $a_{1} \cup b=a \cup b \supset \mathfrak{q}$. Also $a_{1} \supset \mathfrak{q}$ since otherwise $a_{1}^{\prime} \supset \mathfrak{q}$. Now $\mathfrak{q} \supset\left(a_{1} \cap b\right)$ $\cap q \supset a \cap b$ and $q \neq\left(a_{1} \cap b\right) \cap q$ since otherwise $b \supset q$. Hence $q>a \cap b=\left(a_{1} \cap b\right)$ $\cap q$. By the Birkhoff condition we have $\mathfrak{q}_{1}=q \cup\left(a_{1} \cap b\right)>a_{1} \cap b$. Since $a_{1} \cup b \supset \mathfrak{q}$ we have $a_{1} \cup b \supset q_{1}>a_{1} \cap b$. Clearly $a_{1} D q_{1}, b \supset q_{1}$, and $a_{1} \neq a$. This contradicts the maximal property of $a$. Hence we have $\mathfrak{p} \cup a \supset \mathfrak{q}$.

Now let $a \supset p_{1}>a \cap b . p_{1} \neq q$ since otherwise $a \supset q$ and $\mathfrak{p}_{1} \neq \mathfrak{p}$ since otherwise $a \cap b \supset \mathfrak{p}>a \cap b$. Hence $\mathfrak{p}_{1} \cup \mathfrak{p} D \mathfrak{q}$ since every three ideals covering $a \cap b$ generate a Boolean algebra. Thus $p_{1} \cup p D q$ for some $p_{1} \in p_{1}$. Set $x=p_{1} \cap a$. Then

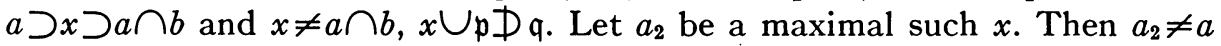
since $a \cup \mathfrak{p} \supset \mathfrak{q}$. Hence $a \supset \mathfrak{p}_{2}>a_{2}$ for some ideal $\mathfrak{p}_{2}$. Then if $\mathfrak{p}_{2} \cup \mathfrak{p} D \mathfrak{q}$ we have $p_{2} \cup p D q$ for some $p_{2} \in p_{2}$. Let $a_{2}^{\prime}=a \cap p_{2}$. Then $a \supset a_{2}^{\prime} \supset a \cap b, a_{2}^{\prime} \neq a \cap b$ and $a_{2}^{\prime} \cup \mathfrak{p} D \mathfrak{q}$ contrary to the maximal property of $a_{2}$. Hence $p_{2} \cup \mathfrak{p} \supset \mathfrak{q}$. Let $\mathfrak{q}_{2}=a_{2} \cup \mathfrak{q}$ and $\mathfrak{p}_{3}=a_{2} \cup \mathfrak{p}$. We have $a_{2} D \mathfrak{p}$ since $a \perp \mathfrak{p}$ and hence $\mathfrak{p}_{2}, \mathfrak{q}_{2}, \mathfrak{p}_{3}>a_{2}$ by the Birkhoff condition. Clearly $\mathfrak{p}_{2} \cup \mathfrak{p}_{3}=\mathfrak{p}_{2} \cup a_{2} \cup \mathfrak{p} \supset a_{2} \cup \mathfrak{q}=\mathfrak{q}_{2}$. Now $\mathfrak{p}_{2} \neq \mathfrak{q}_{2}$ since otherwise $a \supset q$. Also $q_{2} \neq p_{3}$ since otherwise $a_{2} \cup p \supset q$ contrary to the definition of $a_{2}$. Finally $\mathfrak{p}_{2} \neq \mathfrak{p}_{3}$ since $a \nsupseteq \mathfrak{p}$. Thus $\mathfrak{p}_{2}, \mathfrak{q}_{2}, \mathfrak{p}_{3}$ do not generate a Boolean algebra, which contradicts our hypothesis. Hence either $a \supset \mathfrak{q}$ or $b \supset \mathfrak{q}$.

On the other hand if three ideals $\mathfrak{a}, \mathfrak{b}, \mathfrak{c}$ covering $d$ do not generate a Boolean algebra, then $\mathfrak{a} \cup \mathfrak{b} \supset \mathfrak{c}$ say. Since $d=\mathfrak{a} \cap \mathfrak{b}$, elements $a \in \mathfrak{a}$ and $b \in \mathfrak{b}$ 
exist such that $d=a \cap b$. Hence $a \cup b \supset \mathfrak{a} \cup \mathfrak{b} \supset \mathfrak{c}>a \cap b=d$. If $a \supset \mathfrak{c}$, then $a \supset \mathfrak{a} \cup \mathfrak{c} \supset \mathfrak{b}$ and $d=a \cap b \supset \mathfrak{b}$ which is impossible. Hence $a \supset \mathfrak{c}$ and $b \supset \mathfrak{c}$. Thus $a \cup b \supset \mathfrak{c}>a \cap b$ but $a \nsupseteq \mathrm{c}$ and $b \perp c$. This completes the proof of the theorem.

We show now that if the ascending chain condition holds and the condition of Theorem 6.3 is satisfied, then $E_{5}$ is equivalent to the Birkhoff condition. A preliminary lemma is required.

LEmma 6.1. $\subseteq$ is a Birkhoff lattice if and only if $b \supset a, b D \mathfrak{p}>a$ implies $\mathfrak{p} \cup b>b$.

The necessity of the condition is obvious. To prove the sufficiency let $\mathfrak{b}>a$, $\mathfrak{c} \supset a$ and $\mathfrak{c} D \mathfrak{b}$. If $\mathfrak{b} \cup \mathfrak{c} \ngtr \mathfrak{c}$ we have $\mathfrak{b} \cup \mathfrak{c} \supset \mathfrak{b} \supset \mathfrak{c}$ where $\mathfrak{b} \cup \mathfrak{c} \neq \mathfrak{b} \neq \mathfrak{c}$. Since $\mathfrak{b} D \mathfrak{b}$ an element $d \in \mathfrak{b}$ exists such that $d \Phi \mathfrak{b}$. Since $d \supset \mathfrak{c}$ and $\mathfrak{c} D \mathfrak{d}$ there exists an element $c \in \mathfrak{c}$ such that $d \supset c$ and $c \nsupseteq \mathfrak{b}$. Now $c \supset a, c D \mathfrak{b}>a$. Hence $c \cup \mathfrak{b}>c$. But $c \cup \mathfrak{b} \supset d \cap(c \cup \mathfrak{b}) \supset c$ and $c \cup \mathfrak{b} \neq d \cap(c \cup \mathfrak{b})$ since $d D \mathfrak{b}$. Thus $c=d \cap(c \cup \mathfrak{b})$ $\supset \mathfrak{b} \cap(\mathfrak{c} \cup \mathfrak{b})=\mathfrak{d}$ which contradicts $c \triangleright D \mathfrak{b}$. Hence $\mathfrak{b} \cup \mathfrak{c}>\mathfrak{c}$ and $\mathfrak{S}$ is a Birkhoff lattice.

ThEOREM 6.4. Let $\subseteq$ satisfy $\mathrm{E}_{5}$, the ascending chain condition, and let $a \cup b \supset \mathfrak{q}>a \cap b$ imply $a \supset \mathfrak{q}$ or $b \supset \mathfrak{q}$. Then $\subseteq$ is a Birkhoff lattice.

Proof. Let $X$ be the set of all elements $x$ such that $y \supset x, \mathfrak{p}>x, y \supset \mathfrak{p}$, and $\mathfrak{p} \cup y \ngtr y$ for some $y$ and $\mathfrak{p}$. If $\subseteq$ is not a Birkhoff lattice, then $X$ is non-empty by Lemma 6.1. Let $a$ be a maximal element of $X$. Then $b$ and $p$ exist such that $b \supset a, b \supset \mathfrak{p}>a$ and $b \cup \mathfrak{p} \supset \mathfrak{c}>b, b \cup \mathfrak{p} \neq \mathfrak{c}$. Now $\mathfrak{p} \supset \mathfrak{p} \cap \mathfrak{c} \supset a$ and $\mathfrak{p} \neq \mathfrak{p} \cap \mathfrak{c}$ since otherwise $b \cup \mathfrak{p}=\mathfrak{c}$. Hence $\mathfrak{p} \cap \mathfrak{c}=a$. Let $p \in \mathfrak{p}, c \in \mathfrak{c}$ such that $p \cap c=a$. Then $p \cap c=p \cap b=a$ and $c \neq b$. Hence by $E_{5}$ an element $p_{1}$ exists such that $p \supset p_{1} \supset a$, $p_{1} \neq a$ and $b=c \cap\left(b \cup p_{1}\right)$. Now $b \cup p_{1} \perp c$ since otherwise $b=c \cap\left(b \cup p_{1}\right) \supset c$ which contradicts $c>b$. Now suppose that we have found $p_{1}, \cdots, p_{k}$ such that $b \cup p_{1} \cup p_{2} \cup \ldots \cup p_{k} D c$ and $\left(b \cup p_{1} \cup \ldots \cup{p_{i}}_{i} \cap p_{i+1}=a(i=1, \cdots, k-1)\right.$. Since $b \cup p_{1} \cup p_{2} \cup \ldots \cup p_{k} D \mathfrak{c}$ we have $b \cup p_{1} \cup \ldots \cup_{p_{k}} D_{\mathfrak{p}}$ and hence $\left(b \cup p_{1} \cup \ldots \cup p_{k}\right) \cap \mathfrak{p}=a$. Thus $p_{k+1}^{\prime} \in \mathfrak{p}$ exists such that $\left(b \cup p_{1} \cup \ldots \cup p_{k}\right)$ $\cap p_{k+1}^{\prime}=a$. Let $p_{k+1}^{\prime \prime}=p_{k+1}^{\prime} \cap p$. Then $\left(b \cup p_{1} \cup \ldots \cup p_{k}\right) \cap p_{k+1}^{\prime \prime}=a$ and $c \cap p_{k+1}$ $=b \cap p_{k+1}^{\prime \prime}=a, \quad p_{k+1}^{\prime \prime} \neq a$. Hence by $\mathrm{E}_{5}$ an element $p_{k+1}$ exists such that $p_{k+1}^{\prime \prime} \supset p_{k+1} \supset a, p_{k+1} \neq a$ and $b=c \cap\left(b \cup p_{k+1}\right)$. Then $b \cup p_{k+1} D c$ since otherwise $b=c \cap\left(b \cup p_{k+1}\right) \supset \mathfrak{c}$. Now $c \supset \mathfrak{c} \cap\left(\left(b \cup p_{1} \cup \ldots \cup p_{k}\right) \cap\left(b \cup p_{k+1}\right)\right) \supset b$ and $\mathfrak{c} \neq$ $\mathfrak{c} \cap\left(\left(b \cup p_{1} \cup \ldots \cup \cup_{p_{k}}\right) \cap\left(b \cup p_{p_{k+1}}\right)\right)$ since otherwise $b \cup p_{k+1} \supset\left(b \cup p_{1} \cup \ldots\right.$ $\left.\cup_{p_{k}}\right) \cap\left(b \cup p_{k+1}\right) \supset c$. Hence since $c>b$ we have $c>c \cap\left(\left(b \cup p_{1} \cup \ldots \cup p_{k}\right)\right.$ $\left.\cap\left(b \cup p_{k+1}\right)\right)=b$. But now since $b$ is a proper divisor of $a$, by the maximal property of $a$ we must have $c \cup\left(\left(b \cup p_{1} \cup \ldots \cup p_{k}\right) \cap\left(b \cup_{p_{k+1}}\right)\right)>\left(b \cup p_{1}\right.$ $\left.\cup \ldots \cup \mathcal{p}_{k}\right) \cap\left(b \cup p_{k+1}\right)$. Now suppose that $b \cup p_{1} \cup \ldots \cup \cup_{p_{k+1}} \supset c$. Then $\left(b \cup p_{1} \cup \ldots \cup p_{k}\right) \cup\left(b \cup p_{k+1}\right) \supset c \cup\left(\left(b \cup p_{1} \cup \ldots \cup p_{k}\right) \cap\left(b \cup p_{k+1}\right)\right)>\left(b \cup p_{1}\right.$ $\left.\cup \ldots \cup p_{k}\right) \cap\left(b \cup p_{k+1}\right)$. Hence by hypothesis either $b \cup_{p_{1}} \cup \ldots \cup \cup_{p_{k}} \supset \mathfrak{c}$ or $b \cup p_{k+1} \supset \mathrm{c}$ both of which are impossible. Thus $b \cup p_{1} \cup \ldots \cup p_{k+1} D$ c. By induction we get an infinite chain $b \subset b \cup{p_{1}}_{1} \subset b \cup \cup_{p_{1}} \cup_{p_{2}} \subset \cdots \subset b \cup{p_{1}}_{1} \cup \cdots$ 
$\cup_{p_{i}} \subset \cdots$ and $b \cup p_{1} \cup \cdots \cup \cup_{p_{i}} \neq b \cup \cup_{p_{1}} \cup \cdots \cup_{p_{i+1}}$ since $\left(b \cup p_{1} \cup \ldots\right.$ $\cup_{p_{i}} \cap \cap p_{i+1}=a$. This chain contradicts the ascending chain condition and hence $\subseteq$ is a Birkhoff lattice.

The condition $a \cup b \supset \mathfrak{q}>a \cap b$ implies $a \supset \mathfrak{q}$ or $b \supset \mathfrak{q}$, may be given a purely combinatorial statement as follows:

THEOREM 6.5. $a \cup b \supset \mathfrak{q}>a \cap b$ implies $a \supset \mathfrak{q}$ or $b \supset \mathfrak{q}$ if and only if

(A) $a \cup b \supset x \supset a \cap b, a \cap x=b \cap x=a \cap b$ implies $x=a \cap b$.

Proof. Let $a \cup b \supset x \supset a \cap b, a \cap x=b \cap x=a \cap b$. If $x \neq a \cap b$, let $x \supset \mathfrak{q}>a \cap b$. Then $a \cup b \supset \mathfrak{q}>a \cap b$ and hence $a \supset \mathfrak{q}$ say. But then $a \cap b=a \cap x \supset \mathfrak{q}>a \cap b$ which is impossible. Hence $x=a \cap b$.

On the other hand, let $a \cup b \supset \mathfrak{q}>a \cap b$. If $a \nsupseteq q$ and $b D q$ we have $a \cap q$ $=b \cap q=a \cap b$. Hence for some $x \in \mathfrak{q}$ we have $a \cap x=b \cap x=a \cap b$ and $a \cup b \supset x$ by Theorem 2.2. But then $a \cup b \supset x \supset a \cap b, a \cap x=b \cap x=a \cap b$ and $x \neq a \cap b$ which contradicts condition A.

Lemma 4.6 with Theorems 6.1-6.5 give

THEOREM 6.6. Let $\mathfrak{\subseteq}$ satisfy the ascending chain condition. Then every element of $\mathfrak{S}$ is uniquely expressible as a reduced cross-cut of irreducibles if and only if conditions $\mathrm{E}_{5}$ and $\mathrm{A}$ are satisfied.

Theorem 6.6 has the following interesting corollary:

COROLlARY 6.1. Let $\mathfrak{S}$ satisfy the ascending chain condition and let each element of $\subseteq$ have a unique reduced decomposition into irreducibles. Then a sublattice $\mathfrak{S}^{\prime}$ of $\mathfrak{S}$ has unique irreducible decompositions if and only if $\mathrm{E}_{5}$ holds in $\mathfrak{\varsigma}^{\prime}$.

Axiom A is clearly a slightly stronger form of the requirement that every modular sublattice be distributive. In D1 it was shown that under the assumption of both the ascending and descending chain conditions, this weaker condition and B1 were necessary and sufficient for unique decomposition into irreducibles. But A cannot be replaced by the requirement that every modular sublattice be distributive in Theorem 6.6 as the example of Figure 1 shows.

The non-principal ideals of $\mathfrak{S}$ are the ideals $\mathfrak{a}$, generated by $a_{1}, a_{2}, a_{3}, \cdots$; $\mathfrak{b}$, generated by $b_{1}, b_{2}, b_{3}, \cdots$; and $\mathfrak{c}$, generated by $c_{1}, c_{2}, c_{3}, \cdots$. Clearly $\mathfrak{a}>z$, $\mathfrak{b}>b$, and $\mathfrak{c}>z$. Now $b \cup a_{2 n}=d_{n}>a_{2 n}$ and $b \cup a_{2 n+1}=b_{2 n+1}>a_{2 n+1}$. Hence $b \cup a_{i}$ $>a_{i}$. Similarly $b \cup c_{i}>c_{i}$. Now let $x$ be any element of $\subseteq$ not equal to $b$ or $z$. Then $b_{1} / x$ is an archimedean lattice and $\mathrm{B} 1$ is readily verified in $b_{1} / x$ since each element has at most two covering elements. Thus we have only to verify the Birkhoff condition for non-principal ideals. Clearly $\mathfrak{b}>\mathfrak{a}, b, \mathfrak{c}>z$. Hence $\mathfrak{a} \cup b>b, \mathfrak{a} ; \mathfrak{a} \cup \mathfrak{c}>\mathfrak{a}, \mathfrak{c} ; b \cup \mathfrak{c}>b, \mathfrak{c} . \mathfrak{a} \cup c_{2 n}=e_{n}>c_{2 n}$ and $\mathfrak{a} \cup c_{2 n+1}=b_{2 n+2}>c_{2 n+1}$. Hence $\mathfrak{a} \cup c_{i}>c_{i}$. Similarly $\mathfrak{c} \cup a_{i}>a_{i}$. Thus every element of $\mathfrak{S}$ satisfies the Birkhoff condition in the lattice of ideals and hence $\subseteq$ is a Birkhoff lattice. By Theorem 6.1, $\mathrm{E}_{5}$ holds in $\subseteq$. Now if $\subseteq$ contains a modular, non-distribu- 
tive sublattice it also contains one of the form $\{u, v, w, x, y\}$ where $v \cup w$ $=w \cup x=v \cup x=u$ and $v \cap w=w \cap x=v \cap x=y$. Since every element not equal to $b$ or $z$ is covered by at most two elements we must have $y=z$. But then $v=a_{i}, w=b, x=c_{j}$ and $v \cup w=a_{i} \cup b \neq b \cup c_{j}=w \cup x$ which contradicts $v \cup_{w}$

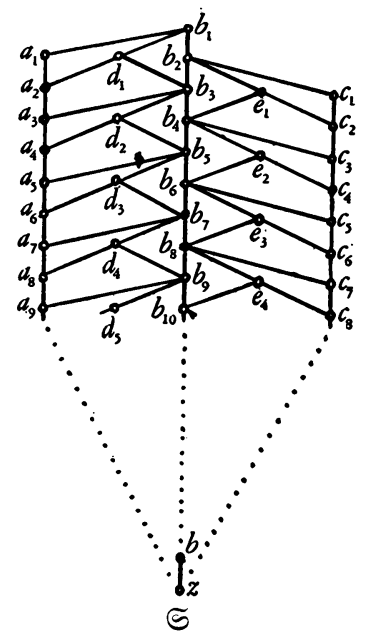

FIG. 1

$=x \cup w$. Hence every modular sublattice of $\subseteq$ is distributive. However $\subseteq$ does not have unique irreducible decompositions since $z=a_{1} \cap b=b \cap c_{1}=a_{1} \cap c_{1}$ and $a_{1}, b, c$ are irreducibles of $\subseteq$. Axiom A does not hold since $a_{i} \cup c_{j} \supset b \supset a_{i} \cap c_{j}$ and $a_{i} \cap b=c_{j} \cap b=a_{i} \cap c_{j}$ but $b \neq a_{i} \cap c_{j}=z$.

Since $a, b, c$ generate a modular lattice, $\mathfrak{l}_{x}$ is modular for every $x$. Hence by Theorem 5.1, the number of components in the reduced decompositions of each element must be unique. This can be readily verified.

According to Theorem 6.2, if every element of a lattice $\subseteq$ is covered by only a finite number of ideals, then $E_{5}$ implies that $\subseteq$ is a Birkhoff lattice. We prove now an even stronger theorem, namely, under this restriction $E_{5}$ implies that B1 holds in the lattice of ideals. We begin with necessary lemmas.

LEMMA 6.2. B1 holds in the lattice of ideals of $\subseteq$ if and only if $\mathfrak{a}>x \cap \mathfrak{a}$ implies $x \cup \mathfrak{a}>x$ for every $\mathfrak{a} \in \mathbb{R}$ and $x \in \subseteq$.

For if $\mathrm{B} 1$ holds in $\mathfrak{R}$, then clearly $\mathfrak{a}>x \cap \mathfrak{a}$ implies $x \cup \mathfrak{a}>x$. Now let $\mathfrak{a}>x \cap \mathfrak{a}$ imply $\mathfrak{a} \cup x>x$ for each $\mathfrak{a}$ and $x$. Suppose that B1 does not hold in $\mathfrak{R}$. Then ideals $\mathfrak{a}$ and $\mathfrak{b}$ exist such that $\mathfrak{a}>\mathfrak{a} \cap \mathfrak{b}$ but $\mathfrak{a} \cup \mathfrak{b} \supset \mathfrak{c} \supset \mathfrak{b}, \mathfrak{a} \cup \mathfrak{b} \neq \mathfrak{c} \neq \mathfrak{b}$. Let $x_{1} \supset \mathfrak{b}, x_{1} \supset \mathfrak{a}$. Such an $x_{1}$ always exists since $\mathfrak{b} D \mathfrak{a}$. Also since $\mathfrak{b} D \mathfrak{c}$, an element $x_{2}$ exists such that $x_{2} \supset \mathfrak{b}, x_{2} D \mathfrak{c}$. Finally since $\mathfrak{b} \cup \mathfrak{c} D \mathfrak{a}$ there is an element $x_{3}$ such that $x_{3} \supset \mathfrak{b}, x_{3} \cup \mathfrak{c} D \mathfrak{a}$. Let $x=x_{1} \cap x_{2} \cap x_{3}$. Then $x \supset \mathfrak{b}, x D \mathfrak{a}$, and $x \cup \mathfrak{c} D \mathfrak{a}$. Now $\mathfrak{a} \supset \mathfrak{a} \cap x \supset \mathfrak{a} \cap \mathfrak{b}$ and $\mathfrak{a} \neq \mathfrak{a} \cap x$. Hence $\mathfrak{a} \cap x=\mathfrak{a} \cap \mathfrak{b}$ and thus $\mathfrak{a}>\mathfrak{a} \cap x$. By 
hypothesis then $x \cup_{\mathfrak{a}}>x$. Now $x \cup_{\mathfrak{a}} \supset \mathfrak{c} \cup_{x} \supset x$ and $\mathfrak{c} \cup x \neq x$. Hence $x \cup_{\mathfrak{a}}=\mathfrak{c} \cup x$ which implies $x \cup \mathfrak{c} \supset \mathfrak{a}$ contrary to the definition of $x$. Hence B1 holds in $\mathbb{R}$.

Leмma 6.3. Let $\subseteq$ be a Birkhoff lattice. Then if $\mathfrak{a}>x \cap \mathfrak{a}$ and $x \cup \mathfrak{a} \ngtr x$, each $x \cap a, a \in \mathfrak{a}$ is covered by an infinite number of ideals.

For let $\mathfrak{a}>x \cap \mathfrak{a}$ and $x \cup \mathfrak{a} \ngtr x$. Then clearly $a \cap x \neq a$ for every $a \in \mathfrak{a}$ since otherwise $x \supset a \supset \mathfrak{a}$ and $\mathfrak{a} \ngtr x \cap \mathfrak{a}$. Hence $a \supset \mathfrak{p}_{a}>a \cap x$ for some ideal $\mathfrak{p}_{a}$ by Theorem 2.1. Let $S_{a}$ denote the set of all ideals $\mathfrak{p}_{a}$. Now $x \supset \mathfrak{p}_{a}$, since otherwise $a \cap x \supset \mathfrak{p}_{a}>a \cap x$ which is impossible. Thus $\mathfrak{p}_{a} \supset x \cap \mathfrak{p}_{a} \supset a \cap x$ and $\mathfrak{p}_{a} \neq x \cap \mathfrak{p}_{a}$. Hence $x \cap \mathfrak{p}_{a}=a \cap x$ and $\mathfrak{p}_{a}>x \cap \mathfrak{p}_{a}$ where $x \cap \mathfrak{p}_{a}$ is a principal ideal of $\mathbb{R}$. Since $\subseteq$ is a Birkhoff lattice we have $x \cup \mathfrak{p}_{a}>x$ for every $\mathfrak{p}_{a}$.

Now in $S_{a}$ we set $\mathfrak{p}_{a} \sim \mathfrak{p}_{a}^{\prime}$ if and only if $x \cup \mathfrak{p}_{a}=x \cup \mathfrak{p}_{a}^{\prime}$. Then $\sim$ is an equivalence relation which separates $S_{a}$ into mutually exclusive sets of ideals. Let $B_{a}$ denote an arbitrary equivalence class and let $\mathfrak{b}_{a}=\Sigma\left(B_{a}\right)$. If $\mathfrak{p}_{a} \in B_{a}$, then $x \cup \mathfrak{p}_{a}=x \cup \mathfrak{p}_{a}^{\prime}$ for every other ideal $\mathfrak{p}_{a}^{\prime}$ of $B_{a}$ and hence $x \cup \mathfrak{p}_{a}=x \cup \mathfrak{b}_{a}$. Thus $x \cup \mathfrak{b}_{a}>x$. Let $T_{a}$ denote the set of ideals $\mathfrak{b}_{a}$. Now if $a \supset a_{1} \supset \mathfrak{a}$ and $\mathfrak{b}_{a_{1}} \in T_{a_{1}}$, let $\mathfrak{b}_{a_{1}} \supset \mathfrak{p}_{a_{1}}>x \cap a_{1}$. Then $\mathfrak{p}_{a_{1}} \supset(x \cap a) \cap \mathfrak{p}_{a_{1}} \supset x \cap a_{1}$ and $\mathfrak{p}_{a_{1}} \neq(x \cap a) \cap \mathfrak{p}_{a_{1}}$ since otherwise $x \supset x \cap a \supset \mathfrak{p}_{a_{1}}$ which contradicts $x D \mathfrak{p}_{a_{1}}$. Hence $\mathfrak{p}_{a_{1}}>(x \cap a) \cap \mathfrak{p}_{a_{1}}$ $=x \cap a_{1}$ and $(x \cap a) \cup \mathfrak{p}_{a_{1}}>x \cap a$ by the Birkhoff condition. Also $a \supset a \cup a_{1}$ $\supset(x \cap a) \cup \mathfrak{p}_{a_{1}}>x \cap a$ and hence $\mathfrak{p}_{a}=(x \cap a) \cup \mathfrak{p}_{a_{1}}$ belongs to $S_{a}$. Now $x \cup \mathfrak{p}_{a}$ $=x \cup(x \cap a) \cup \mathfrak{p}_{a_{1}}=x \cup_{\mathfrak{p}_{a_{1}}}$. Let $\mathfrak{b}_{a_{1}} \supset \mathfrak{p}_{a_{1}}^{\prime}>x \cap a_{1}$. Then $x \cup_{\mathfrak{p}_{a}^{\prime}}^{\prime}=x \cup_{\mathfrak{p}_{a_{1}}^{\prime}}$ where $\mathfrak{p}_{a}^{\prime}=(x \cap a) \cup \mathfrak{p}_{a_{1}}^{\prime}$. Thus $x \cup \mathfrak{p}_{a}=x \cup \mathfrak{p}_{a_{1}}=x \cup \mathfrak{p}_{a_{1}}^{\prime}=x \cup \mathfrak{p}_{a}^{\prime}$ and $\mathfrak{p}_{a} \sim \mathfrak{p}_{a}^{\prime}$ in $S_{a}$. Hence $\mathfrak{b}_{a_{1}} \subset \mathfrak{b}_{a}$ where $\mathfrak{b}_{a}$ is an ideal of $T_{a}$. Now suppose that $T_{a_{1}}$ contains a second ideal $\mathfrak{b}_{a_{1}}^{\prime}$, which is divisible by $\mathfrak{b}_{a}$. Let $\mathfrak{b}_{a_{1}}^{\prime} \supset \mathfrak{p}_{a_{1}}^{\prime \prime}>x \cap a_{1}$. Then $x \cup \mathfrak{p}_{a_{1}}=x \cup \mathfrak{p}_{a}=x \cup \mathfrak{b}_{a} \supset x \cup \mathfrak{b}_{a_{1}}^{\prime} \supset x \cup \mathfrak{p}_{a_{1}}^{\prime \prime} \supset x$. Since $x \cup \mathfrak{p}_{a_{1}}^{\prime \prime} \neq x$, we have $x \cup \mathfrak{p}_{a_{1}}$ $=x \cup \mathfrak{p}_{a_{1}}^{\prime \prime}$ and $\mathfrak{p}_{a_{1}} \sim \mathfrak{p}_{a_{1}}^{\prime \prime}$ in $S_{a_{1}}$ contrary to assumption. Hence $\mathfrak{b}_{a_{1}}$ is the only ideal of $T_{a_{1}}$ divisible by $\mathfrak{b}_{a}$. Next suppose that $T_{a}$ contains another ideal $\mathfrak{b}_{a}^{\prime}$ such that $\mathfrak{b}_{a}^{\prime} \supset \mathfrak{b}_{a_{1}}$. Then $x \cup \mathfrak{b}_{a}^{\prime} \supset x \cup \mathfrak{b}_{a_{1}}=x \cup \mathfrak{b}_{a}$ and hence $\mathfrak{b}_{a}=\mathfrak{b}_{a}{ }^{\prime}$. We thus conclude that each ideal $\mathfrak{b}_{a_{1}}$ of $T_{a_{1}}$ is divisible by exactly one ideal $\mathfrak{b}_{a}$ of $T_{a}$ and $\mathfrak{b}_{a_{1}}$ is the only ideal of $T_{a_{1}}$ which is divisible by $\mathfrak{b}_{a}$.

Let $n_{a}$ denote the cardinal number of the set $T_{a}$. If $x \cap a$ is covered by only a finite number of ideals for some $a$, then $n_{a}$ is finite for some $a$ and hence has a minimal value for some $a_{0}$. If $a_{0} \supset a \supset \mathfrak{a}$, then $n_{a} \leqq n_{a_{0}}$ since distinct ideals of $T_{a}$ are divisible by distinct ideals of $T_{a_{0}}$. But since $n_{a_{0}}$ is minimal we have $n_{a}=n_{a_{0}}$. Hence each ideal of $T_{a_{0}}$ divides exactly one ideal of $T_{a}$. Let $\mathfrak{b}_{0}$ be an ideal of $T_{a_{0}}$ and let $\mathfrak{b}_{a}$ denote the ideal of $T_{a}$ divisible by $\mathfrak{b}_{0}$. In general, for any $a \in \mathfrak{a}$, let $\mathfrak{b}_{a}$ be the ideal of $T_{a}$ divisible by $x \cup \mathfrak{b}_{0}$. Such an ideal $\mathfrak{b}_{a}$ always exists since $\mathfrak{b}_{0} \supset \mathfrak{b}_{a}^{\prime}$ where $\mathfrak{b}_{a}^{\prime} \in T_{a n a_{0}}$ and hence $\mathfrak{b}_{a} \in T_{a}$ exists such that $\mathfrak{b}_{a} \supset \mathfrak{b}_{a}{ }^{\prime}$. Clearly $x \cup \mathfrak{b}_{0}=x \cup \mathfrak{b}_{a}{ }^{\prime}=x \cup \mathfrak{b}_{a} \supset \mathfrak{b}_{a}$ and $\mathfrak{b}_{a}$ is unique as shown above. If $a \supset a^{\prime}$, we have $\mathfrak{b}_{a} \supset \mathfrak{b}_{a^{\prime}}$ since $x \cup \mathfrak{b}_{a}=x \cup \mathfrak{b}_{a^{\prime}}$. Hence $\mathfrak{b}_{a_{1}} \cap \mathfrak{b}_{a_{2}} \cap \cdots \cap \mathfrak{b}_{a_{n}}$ $\supset \mathfrak{b}_{a_{1} \cap a_{2} \cap \cdots n a_{n}}$.

Now let $\mathfrak{a}_{1}=\prod_{a \in \mathfrak{a}}\left(\mathfrak{b}_{a}\right)$. Since $a \supset \mathfrak{b}_{a} \supset \mathfrak{a}_{1}$, we have $\mathfrak{a} \supset \mathfrak{a}_{1} \supset x \cap \mathfrak{a}$. If $\mathfrak{a}_{1}=x \cap \mathfrak{a}_{1}$ then $x \cap a$ divides the cross-cut of a finite number of the ideals $\mathfrak{b}_{a}$ and hence 
$x \cap a \supset \mathfrak{b}_{a^{\prime}}$, for some $a^{\prime}$. But then $(x \cap a) \cap a^{\prime} \supset \mathfrak{b}_{a^{\prime}} \cap \mathfrak{b}_{a^{\prime}} \supset \mathfrak{b}_{a^{\prime}} \supset \mathfrak{b}_{a n a^{\prime}} \supset x \cap\left(a \cap a^{\prime}\right)$ and $\mathfrak{b}_{a n a^{\prime}}=x \cap\left(a \cap a^{\prime}\right)$ contrary to the definition of $\mathfrak{b}_{a n a^{\prime}}$. Thus $\mathfrak{a}_{1} \neq x \cap \mathfrak{a}$ and hence $\mathfrak{a}=\mathfrak{a}_{1}$ since $\mathfrak{a}>x \cap \mathfrak{a}$. But then $x \cup \mathfrak{b}_{0} \supset x \cup \mathfrak{a}_{1} \supset x \cup \mathfrak{a} \supset x$. Since $x \cup \mathfrak{b}_{0}>x$ and $x D \mathfrak{a}$ we have $x \cup \mathfrak{a}=x \cup \mathfrak{b}_{0}>x$ which contradicts $x \cup \mathfrak{a} \ngtr x$. Hence each

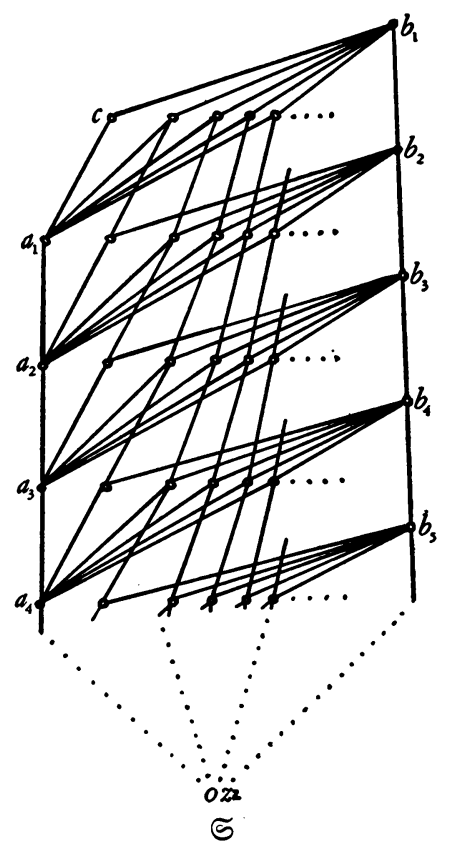

FIG. 2

$x \cap a$ is covered by an infinite number of ideals. The proof is thus complete.

Lemmas 6.2 and 6.3 and Theorem 6.2 give immediately

THEOREM 6.7. Let each element of $\subseteq$ be covered by at most a finite number of ideals. Then the following conditions are equivalent:

(1) $\mathrm{E}_{5}$ holds in $\subseteq$.

(2) $\subseteq$ is a Birkhoff lattice.

(3) B1 holds in the lattice of ideals.

If $\mathfrak{S}$ is a Birkhoff lattice in which each element is not covered by at most a finite number of ideals, then even though the ascending chain condition holds in $\subseteq$ B1 need not be satisfied in the lattice of ideals. For example, consider the lattice diagramed in Figure 2.

All of the elements distinct from $z$ form an ideal $a$ which is generated by $a_{1}, a_{2}, a_{3}, \cdots b_{1}, b_{2}, b_{3}, \cdots$ clearly form an ideal $\mathfrak{b}$ which divides $a$. Now let $\mathfrak{b} \supset \mathfrak{c} \supset \mathfrak{a}, \mathfrak{b} \neq \mathfrak{c}$. Let $y \in \mathfrak{c}, y \notin \mathfrak{b}$. Then there exists a $b_{i}$ such that $b_{i}>x \supset y, x \in \mathfrak{b}$. But by the method of construction there exists an integer $j$ such that 
$x \cap b_{k}=a_{k}$ all $k \geqq j$. Hence $\mathfrak{a} \supset x \cap \mathfrak{b} \supset y \cap \mathfrak{b} \supset \mathfrak{c} \cap \mathfrak{b}=\mathfrak{c}$. Thus $\mathfrak{c}=\mathfrak{a}$ and $\mathfrak{b}>\mathfrak{a}$. Clearly $\mathfrak{b} \cap a_{1}=\mathfrak{a}$ and $\mathfrak{b} \cup a_{1}=b_{1}$. But then $\mathfrak{b}>a_{1} \cap \mathfrak{b}$ and $a_{1} \cup \mathfrak{b} \ngtr a_{1}$. Hence B1 does not hold in $\mathbb{R}$. On the other hand it is readily verified that $\subseteq$ is a Birkhoff lattice since $a$ is the only non-principal ideal which covers a principal ideal and every element distinct from $z$ divides $a$.

The number of ideals covering an element of a lattice is closely related to the number of decompositions of the element into irreducibles. We prove

THEOREM 6.8. Let $\subseteq$ be a Birkhoff lattice in which each element can be represented as a cross-cut of irreducibles. Then if an element a has a finite number of decompositions into irreducibles, $\mathfrak{R}_{a}$ is finite.

Proof. Since $a$ has only a finite number of decompositions into irreducibles, the number of components in the irreducible decompositions of $a$ is bounded. Hence $\Omega_{a}$ is archimedean by Theorem 3.1. Let $p_{1}, \cdots, p_{k}$ be a maximal independent set of point ideals of $\mathfrak{R}_{a}$. Let $\mathbb{B}_{i}=\mathfrak{p}_{1} \cup \ldots \cup \cup_{\mathfrak{p}_{i-1}} \cup \mathfrak{p}_{i+1} \cup \ldots \cup \cup_{\mathfrak{p}_{k}}$ and suppose that $\mathfrak{R}_{a}$ has an infinite sequence $\mathbb{B}_{1}, \cdots, \mathbb{8}_{k}, \mathbb{8}_{k+1}, \cdots$ of simple ideals. Now if for each $i$ there are only a finite number of simple ideals of the sequence which do not divide $\mathfrak{p}_{i}$, we have $\mathbb{B}_{n} \supset \mathfrak{p}_{i}$ for all $n \geqq l_{i}$ for some $l_{i}$. Let $n \geqq \max \left(l_{1}, \cdots, l_{k}\right)$. Then $\mathbb{z}_{n} \supset \mathfrak{p}_{i}, i=1, \cdots, k$, and $\mathfrak{z}_{n} \supset \mathfrak{u}_{a}$, which is impossible. Hence for some $\mathfrak{p}_{i}$, say $\mathfrak{p}_{k}$, there are an infinity of ideals in the sequence $\mathfrak{z}_{1}, \mathfrak{z}_{2}, \cdots$ which do not divide $\mathfrak{p}_{k}$. We may assume that $z_{k}, \mathbb{z}_{k+1}, \cdots$ do not divide $\mathfrak{p}_{k}$. Let $q_{i} \supset \mathbb{z}_{i}, q_{i} \supset \mathfrak{u}_{a}$. Then $q_{i} \neq q_{j}, i \neq j$, since otherwise $q_{i}=q_{i} \cup q_{j}$ $\supset \mathfrak{z}_{i} \cup \mathfrak{z}_{j} \supset \mathfrak{u}_{a}$. Now $a=\mathfrak{p}_{k} \cap \mathbb{z}_{k+l}=\mathfrak{z}_{1} \cap \cdots \cap \mathfrak{z}_{k-1} \cap \mathbb{z}_{k+l}(l=0,1,2, \cdots)$ implies $a=q_{1} \cap q_{2} \cap \cdots \cap q_{k-1} \cap q_{k+l}(l=0,1,2, \cdots)$ by Theorem 3.3. If this representation is reduced for each $l, q_{k+l}$ always remains since otherwise $a=q_{1} \cap \cdots \cap q_{k-1} \supset \mathfrak{p}_{k}$. Hence $a$ has an infinite number of irreducible decompositions, which contradicts our hypothesis. Thus $\ell_{a}$ has only a finite number of simple ideals. But by Theorem 3.2 every ideal of $\mathfrak{R}_{a}$ can be expressed as a cross-cut of simple ideals. Hence $\mathfrak{R}_{a}$ is finite.

Theorems 6.7 and 6.8 give

THEOREM 6.9. Let $\mathfrak{S}$ be a lattice in which every element has at least one and at most a finite number of decompositions into irreducibles. Then $\Im$ is a Birkhoff lattice if and only if B1 is satisfied in the lattice of ideals $\left({ }^{11}\right)$.

7. Example of a Birkhoff lattice. In $\$ 3$ we have shown that the existence of a decomposition into irreducibles for an element $a$ of a modular lattice implies that $\mathfrak{R}_{a}$ is archimedean. Hence if the ascending chain condition holds,

(11) Various considerations suggest that the finiteness of the number of irreducible decompositions of an element always implies the finiteness of the number of ideals covering the element, in which case $E_{5}$ and the finiteness of the number of decompositions would imply that $\subseteq$ is a Birkhoff lattice. However, I have been unable to prove this. I have also been unable to prove that $E_{5}$ is equivalent to the Birkhoff condition under the assumption of the ascending chain condition although this seems quite likely. 
$\mathfrak{R}_{a}$ is archimedean for each $a$. Also if the ascending chain condition holds in a Birkhoff lattice and the number of components is bounded for an element $a$, then $\mathfrak{R}_{a}$ is archimedean. We shall construct in this section a Birkhoff lattice satisfying the ascending chain condition but containing an element $a$ such that $\mathfrak{R}_{a}$ is not archimedean. By the above remark the number of components in the irreducible decompositions of $a$ must be unbounded.

Latin capitals $A, B, C, \cdots$ will denote finite subsets of the set of positive integers $1,2,3, \cdots$. If $A$ is such a set, let $n(A)$ denote the number of elements in $A$. Small Latin letters $a, b, c, \cdots$ will denote positive integers. $A \cup B$ and $A \cap B$ will denote set-theoretic union and cross-cut respectively and $a \cup b$, $a \cap b$ are respectively the maximum and minimum of $a$ and $b$. Let $\subseteq$ be the set of all ordered couples $\alpha=\{A, a\}$ where $n(A)<a$ together with the elements $u$ and $z$. In $\subseteq$ we define

$$
\begin{aligned}
& \alpha \cup \beta=\{A \cup B, a \cup b\} \text { if } n(A \cup B)<a \cup b \text {, } \\
& =u \quad \text { if } n(A \cup B) \geqq a \cup b, \\
& \alpha \cap \beta=\{A \cap B, a \cap b\} \text { if } A \cap B \text { is not null, } \\
& =z \quad \text { if } A \cap B \text { is null, } \\
& \alpha \cup z=\alpha \cap u=\alpha, \quad \alpha \cup u=u, \quad \alpha \cap z=z .
\end{aligned}
$$

If $A \cap B$ exists, then $n(A \cap B) \leqq n(A)<a \leqq a \cap b$. Hence $\alpha \cap \beta$ is in $\subseteq$ if $\alpha$ and $\beta$ are in $\subseteq$. Now it can be readily verified that the union and cross-cut so defined in $\mathfrak{S}$ are idempotent, commutative and associative. Consider $\alpha \cap(\alpha \cup \beta)$. If $\alpha \cup \beta=u$, then $\alpha \cap(\alpha \cup \beta)=\alpha$. If $\alpha \cup \beta \neq u$, then $\alpha \cap(\alpha \cup \beta)$ $=\{A \cap(A \cup B), a \cap(a \cup b)\}=\{A, a\}=\alpha$. Hence $\alpha \cap(\alpha \cup \beta)=\alpha$ in all cases. Similarly $\alpha \cup(\alpha \cap \beta)=\alpha$. Hence $\subseteq$ is a lattice under the union and cross-cut operations defined above. Clearly $\alpha \supset \beta$ if and only if $A \supset B$ and $a \leqq b$.

$\subseteq$ satisfies the ascending chain condition. For let $\alpha_{1} \subset \alpha_{2} \subset \alpha_{3} \subset \cdots$ be an infinite ascending chain. We may assume that $\alpha_{1} \neq z$ so that $\alpha_{1}=\left\{A_{1}, a_{1}\right\}$. But then $A_{1} \subset A_{2} \subset A_{3} \subset \cdots$ and $a_{1} \geqq a_{2} \geqq a_{3} \geqq \cdots$. Now $n\left(A_{i}\right)<a_{i} \leqq a_{1}$. Hence the chain $A_{1} \subset A_{2} \subset \cdots$ has only a finite number of distinct sets. Clearly the chain $a_{1} \geqq a_{2} \geqq \cdots$ has only a finite number of distinct members. Thus the chain $\alpha_{1} \subset \alpha_{2} \subset \ldots$ has only a finite number of distinct members.

Now let $a$ be an ideal of $\mathfrak{S}$ with elements $\beta, \gamma, \delta, \cdots$. Then the set of integers is either bounded or unbounded. If bounded, let $a$ be the largest of them. Now suppose that $B \cap C \cap D \cap \ldots$ is null. Then there exist a finite number of them $B, C, \cdots, L$ whose cross-cut is null. But then $a$ contains $z$ since $\mathfrak{a}$ is closed with respect to finite cross-cut. Hence $\mathfrak{a}=\mathfrak{S}$ in this case. If $B \cap C \cap D \cap \ldots$ is not null, let $A=B \cap C \cap D \cap \ldots$. Then $A=B \cap C \cap \cdots \cap L$ for a finite number of the sets and hence $\alpha^{\prime}=\left\{A, a^{\prime}\right\}$ and $\alpha^{\prime \prime}=\left\{A^{\prime}, a\right\}$ are in a. But then a contains $\alpha=\alpha^{\prime} \cap \alpha^{\prime \prime}=\{A, a\}$ and $\beta \supset \alpha$ for every $\beta \in \mathfrak{a}$. Hence if the integers of the ideal are bounded, $\mathfrak{a}$ is a principal ideal. If the integers of the ideal are unbounded, then as before either $\mathfrak{a}=\mathfrak{S}$ 
or there exists a set $A$ such that $\{A, a\}$ is in $a$ for each positive integer $a$ and $\beta \supset\{A, a\}$ for some $a$ if $\beta \in \mathfrak{a}$. Hence the ideals of $\subseteq$ have the form $\mathfrak{a}=\{A, \infty\}$ if $a$ is not principal. $\infty$ denotes the ideal of all positive integers. Clearly if $a$ and $b$ are positive integers or $\infty$

$$
\begin{aligned}
& \mathfrak{a} \cap \mathfrak{b}=\{A \cap B, a \cap b\} \text { if } A \cap B \text { is not null, } \\
& =z \quad \text { if } A \cap B \text { is null, } \\
& \mathfrak{a} \cup \mathfrak{b}=\{A \cup B, a \cup b\} \text { if } n(A \cup B)<a \cup b \text {, } \\
& =u \quad \text { if } n(A \cup B) \geqq a \cup b \text {. }
\end{aligned}
$$

Theorem 7.1. $\subseteq$ is a Birkhoff lattice.

Proof. We shall show that B1 holds in the lattice of ideals. Let $\mathfrak{a}>\mathfrak{a} \cap \mathfrak{b}$. If $\mathfrak{a} \cap \mathfrak{b}=z$, then $A \cap B$ is null. Since $\mathfrak{a}>z$ we have $\mathfrak{a}=\{(a), \infty\}$. But then $\mathfrak{a} \cup \mathfrak{b}=\{(a) \cup B, \infty \cup b\}=\{(a) \cup B, b\}$ and $(a) \cup B>B$. Thus $\mathfrak{a} \cup \mathfrak{b}>\mathfrak{b}$. If $\mathfrak{a} \cap \mathfrak{b} \neq z$, then $\{A, a\}>\{A \cap B, a \cap b\}$ and hence either $A>A \cap B, a=a \cap b$ or $A=A \cap B, a=(a \cap b)-1$. In the first case $A>A \cap B \rightarrow A \cup B>B$ and $a=a \cap b$ implies $b=a \cup b$. Hence $\mathfrak{a} \cup \mathfrak{b}=\{A \cup B, a \cup b\}=\{A \cup B, b\}>\{B, b\}=\mathfrak{b}$. If $n(A \cup B) \geqq b$, then $n(B)=b-1$ and hence $u>B$. In the second case $A=A \cap B$ $\rightarrow A \cup B=B$ and $a>a \cap b \rightarrow a=b-1$. Hence $\mathfrak{a} \cup \mathfrak{b}=\{B, a\}>\mathfrak{b}$. Thus $\mathfrak{a}>\mathfrak{a} \cap \mathfrak{b}$ implies $\mathfrak{a} \cup \mathfrak{b}>\mathfrak{b}$ and $B 1$ holds in the lattice of ideals of $\mathfrak{S}$.

The point ideals of $\mathfrak{R}_{z}$ are clearly the ideals $\mathfrak{p}_{i}=\{(i), \infty\}$. Now $\mathfrak{p}_{1} \cup \mathfrak{p}_{2} \cup \ldots$ $=u$ and hence $\ell_{2}=\mathbb{R}$. The ascending chain $\mathfrak{p}_{1} \subset \mathfrak{p}_{1} \cup \mathfrak{p}_{2} \subset \mathfrak{p}_{1} \cup \mathfrak{p}_{2} \cup \mathfrak{p}_{3} \subset \cdots$ has distinct members and $\mathfrak{R}_{z}$ is thus not archimedean. The point ideals $\mathfrak{p}_{1}, \mathfrak{p}_{2}, \ldots$ are not independent since the union of any infinite set is $u$. However, every finite set of the $\mathfrak{p}_{i}$ is independent and thus generates a Boolean algebra. $\mathfrak{l}_{z}$ is not complemented. For if $\mathfrak{p}_{i} \cup \mathfrak{a}=u$, then $\mathfrak{a}=u$ and $\mathfrak{a} \supset \mathfrak{p}_{i}$. The simple ideals of $\mathfrak{R}_{z}$ are the elements of the form $\{A, a\}$ where $n(A)=a-1$. Clearly $\mathfrak{p}_{i}$ cannot be represented as a cross-cut of simple ideals. Hence it is not true that every ideal of $\mathfrak{R}_{z}$ may be represented as a cross-cut of simple ideals. The irreducibles of $\mathfrak{S}$ are the simple elements of $\mathfrak{R}_{z}$, namely, those elements $\{A, a\}$ with $n(A)=a-1$. Now let $A_{i}$ be the set $\{1,2, \cdots, i-1, i+1, \cdots, k\}(i=1, \cdots, k)$. Then $\alpha_{i}=\left\{A_{i}, k\right\}$ is simple for each $i$ and $z=\alpha_{1} \cap \alpha_{2} \cap \cdots \cap \alpha_{k}$ since $A_{1} \cap A_{2} \cap \cdots \cap A_{k}$ is null. This representation of $z$ is clearly reduced. Hence for any positive integer $k>1, z$ has a reduced decomposition with $k$ components.

This example clearly indicates the complications that may arise if $\mathfrak{R}_{a}$ is not archimedean even though the ascending chain condition holds in $\mathbb{S}$.

8. Example of a lattice satisfying $\mathrm{E}_{5}$ which is not a Birkhoff lattice. Let $S$ be the set of elements $p_{1}, p_{2}, p_{3}, \cdots$. From the set of all subsets of $S$ omit those infinite sets which contain either $p_{1}$ or $p_{2}$ but not both. Denote this set of subsets by $\mathfrak{S}$. $\subseteq$ is clearly closed under infinite cross-cut. Since $\mathfrak{S}$ contains a unit element, the union of any set of sets of $\subseteq$ may be defined in terms 
of the cross-cut operation. $\subseteq$ is thus a continuous lattice in which every element is a union of points. Now consider $A \cup p$ where $A \in \subseteq$ and $p$ is any element of $S$. If $A$ is finite, then clearly $A \cup p=A+p$ where + indicates settheoretic union. Also if $A$ is infinite and contains both $p_{1}$ and $p_{2}, A \cup p=A+p$. Now if $A$ is infinite and does not contain $p_{1}$ or $p_{2}$, then $A \cup p=A+p$ if $p \neq p_{1}, p_{2}$ and $A \cup p=A+p_{1}+p_{2}$ if $p=p_{1}$ or $p=p_{2}$. Now let $A+p_{1}+p_{2} \supset B \supset A$. If $B \neq A$, then $B$ contains $p_{1}$ or $p_{2}$ and hence contains both $p_{1}$ and $p_{2}$ by the definition of $\Im$. Thus $B=A+p_{1}+p_{2}$. Hence in every case $A \cup p>A$ if $p \notin A$.

Now let $A \supset B \supset A \cap C$ and $C \neq A \cap C$ where $A, B, C$ are in $\subseteq$. Since every element of $\subseteq$ is a union of points and $C \neq A \cap C$, there exists a point $p$ such that $C \supset p, A \cap C D p$. Set $C_{1}=(A \cap C) \cup p$. Then $C \supset C_{1} \supset A \cap C$ and $C_{1} \neq A \cap C$. Now $B \cup p \supset A \cap\left(B \cup C_{1}\right) \supset B$ and $B \cup p \neq A \cap\left(B \cup C_{1}\right)$ since otherwise $A \supset B$ $\cup p \supset p$ and $A \cap C \supset p$ which contradicts $A \cap C D p$. Since $B \cup p>B$ we thus have $B=A \cap\left(B \cup C_{1}\right)$ and hence $\mathrm{E}_{5}$ holds in $\subseteq$.

In $\subseteq$ let $\mathfrak{a}$ be the ideal generated by the sets $A_{k}=\left\{p_{k}, p_{k+1}, \cdots\right\}$ $(k=3,4,5, \cdots)$. Then by Theorem 2.1 , there exists a point ideal $p$ such that $\mathfrak{a} \supset \mathfrak{p}>z$. Every set of $\subseteq$ occurring in $\mathfrak{p}$ contains an infinite number of elements. For suppose that $Q \in \mathfrak{p}$ and $Q$ contains only a finite number of elements. Let $k$ be the largest subscript occurring among the elements of $Q$. Then $z=Q \cap A_{k+1} \supset \mathfrak{p} \cap \mathfrak{a} \supset \mathfrak{p}$ which contradicts $\mathfrak{p}>z$. If $Q \in \mathfrak{p}$, then $Q \cup p_{1} \supset p_{2}$ by the definition of $\Im$. Hence $\mathfrak{p} \cup p_{1} \supset p_{1} \cup p_{2} \supset p_{1}$ where $\mathfrak{p} \cup p_{1} \neq p_{1} \cup p_{2}$ and $p_{1} \cup p_{2} \neq p_{1}$. Thus $p \cup p_{1} \ngtr p_{1}$ and hence $\subseteq$ is not a Birkhoff lattice. If $A \supset B$ and $A \neq B$, let $A \supset p, B D p$. Then $A \supset B_{1}>B$ where $B_{1}=B \cup p$. Thus $\subseteq$ is an example of a continuous point lattice in which covering elements exist and $E_{5}$ holds, but which is not a Birkhoff lattice. Whether or not an exchange lattice, i.e., a continuous point lattice satisfying $\mathrm{E}_{5}$ and a finite dependence axiom (Mac Lane [1]) is a Birkhoff lattice is an open question.

\section{G. BIRKHOFF}

\section{REFERENCES}

1. Rings of sets, Duke Mathematical Journal, vol. 3 (1937), pp. 443-454.

2. On combination of subalgebras, Proceedings of the Cambridge Philosophical Society, vol. 29 (1933), pp. 441-464.

R. P. Dilworth

1. Lattices with unique irreducible decompositions, Annals of Mathematics.

2. The arithmetical theory of Birkhoff lattices, submitted to Duke Mathematical Journal.

S. MAC LANE

1. A lattice formulation for transcendence degrees and p-bases, Duke Mathematical Journal, vol. 24 (1938), pp. 455-468.

M. H. STONE

1. The theory of representations of Boolean algebra, these Transactions, vol. 40 (1936), pp. 37-111.

H. Wallman

1. Lattices and topological spaces, Annals of Mathematics, (2), vol. 39 (1938), pp. 112-126.

YALE University,

New Haven, Conn. 